\title{
A local crack-tracking strategy to model three-dimensional crack propagation with embedded methods
}

Chandrasekhar Annavarapu*, Randolph R. Settgast, Efrem Vitali, Joseph P. Morris

Computational Geosciences, Atmospheric, Earth, and Energy Division, Lawrence

Livermore National Laboratory, Livermore, CA 94550, USA

\begin{abstract}
We develop a local, implicit crack tracking approach to propagate embedded failure surfaces in three-dimensions. We build on the global crack-tracking strategy of Oliver et al. (Int. J. Numer. Anal. Meth. Geomech., 2004; 28: 609-632) that tracks all potential failure surfaces in a problem at once by solving a Laplace equation with anisotropic conductivity. We discuss important modifications to this algorithm with a particular emphasis on the effect of the Dirichlet boundary conditions for the Laplace equation on the resultant crack path. Algorithmic and implementational details of the proposed method are provided. Finally, several three-dimensional benchmark problems are studied and results are compared with available literature. The results indicate that the proposed method addresses pathological cases, exhibits better behavior in the presence of closely interacting fractures, and provides a viable strategy to robustly evolve embedded failure surfaces in $3 \mathrm{D}$.
\end{abstract}

Keywords: 3D fracture, embedded cracks, crack-tracking, X-FEM, G-FEM

* Corresponding author: Chandrasekhar Annavarapu, Computational Geosciences, Atmospheric, Earth, and Energy Division, Lawrence Livermore National Laboratory, 7000 East Avenue, L-286, Livermore, CA 94550, USA.

Email address: annavarapusr1@llnl.gov, asc.sekhar@gmail.com

(Chandrasekhar Annavarapu)

Preprint submitted to Comput. Methods Appl. Mech. Engrg.

September 12, 2016

(C) 2016. This manuscript version is made available under the Elsevier user license http://www.elsevier.com/open-access/userlicense/1.0/ 


\section{Introduction}

In recent years, the finite element community has focused attention on a class of embedded interface methods that allow cracks to be oriented arbitrarily with respect to the underlying finite element mesh. Both the Generalized/eXtended Finite Element Methods (G/X-FEM) [1, 2, 3, 4] and the Strong Discontinuity Approaches (also known as embedded FEM or EFEM) $[5,6,7,8]$, facilitate the treatment of cracks as arbitrary interfaces by enhancing the kinematics of the underlying mesh. By treating cracks as arbitrary interfaces, these methods offer the potential for addressing the pathological mesh-dependence of the interface element approaches $[9,10]$. However, since the cracks are now arbitrary with respect to the underlying volume mesh, a tracking mechanism separate from the bulk mesh needs to be introduced to locate the crack surface within the finite element mesh.

Within the embedded finite element methods (G/X-FEM and E-FEM), crack-tracking algorithms can be broadly classified into two major categories: (a) explicit approaches, and (b) implicit approaches. In the explicit methods, cracks are represented as a collection of piecewise segments in 2D, and piecewise triangular and quadrangular surfaces in 3D. Early efforts in this direction discretized the evolving crack surface through a $C^{0}$ continuous surface formed from a union of the triangles and quadrilaterals that separate a cracked tetrahedral element in two (see Areias and Belytschko [11], Gasser and Holzapfel [12]). However, with these approaches in 3D, one needs to modify the normal to the local crack plane in an ad hoc manner if a globally continuous crack path is desired. A more general methodology is to define two completely independent meshes, namely the underlying volume mesh and an independent triangulation of the crack surface (see for e.g. $[13,14,15]$ ). The reader is referred to Garzon et al. [16] and the references therein for a detailed description of these approaches and the current state-of-the-art. While these approaches have the advantage that the element size has no bearing on the accuracy of the crack surface representation, they also require a very specific machinery to handle the computational geometry challenges not available in most general purpose finite element software.

By contrast, in implicit approaches, the crack surface is represented by a zero iso-surface of a signed-distance field associated with the nodes of the underlying finite element mesh. Each crack is represented by two orthogonal level set functions, one associated with the crack surface and the second associated with the crack front such that the intersection of the zero iso-surfaces 
of these two functions precisely locates a crack front (see Möes et al. [17]). In addition, Hamilton-Jacobi type equations are solved over whole or part of the domain using finite difference methods (see Gravouil et al. [18]) or Fast Marching Methods (see Sukumar et al. $[19,20]$ ) to evolve these level set functions as the crack propagates. More recently, improvements to these algorithms have also been proposed in Duflot [21] and Colombo and Massin [22]. Fries et al. [23] have also developed hybrid implicit-explicit approaches that combine the advantages of the aforementioned methods.

In the context of embedded finite element methods, Oliver et al. [24] developed an alternate implicit strategy that solves a Laplace equation with an anisotropic conductivity tensor as a global crack-tracking methodology. In this methodology, all possible crack paths are tracked at once through a nodally defined propagation field. Any given crack then corresponds to an iso-surface value of this field and is easily identified. The idea is remarkably simple yet retains all the advantages associated with the level-set methods and is arguably more suited to being integrated in an existing general purpose finite element framework. Since the original paper by Oliver et al. [24], the approach has been extensively used in both the E-FEM and X-FEM frameworks in several studies (see $[25,26,27,28,29,30,31,32]$ ). In 2D, a comparison between the global and explicit crack tracking approaches is presented in Dumstorff and Meschke [33, 34].

Jäger et al. $[30,31,32]$ in a series of articles present a thorough analysis of the global crack tracking approach when used in conjunction with the phantom node method to model crack propagation in 3D. They compare and contrast the method's performance with the alternatives available and highlight the method's promise to model arbitrary crack propagation problems in 3D. However, they also report that the results of the global approach are sensitive to the Dirichlet boundary conditions applied to the crack-tracking problem. In Jäger et al. [32], they propose geometry-based, and mesh-based strategies to enforce Dirichlet boundary conditions to circumvent this sensitivity.

In the current work, we revisit this sensitivity analysis and demonstrate the spurious behavior that could result for certain choices of Dirichlet boundary conditions for the global crack-tracking method. To resolve this difficulty, we propose an alternative approach that solves the anisotropic Laplace equation in a more localized domain just ahead of the crack front. It is noteworthy that similar ideas have been proposed earlier in Feist and Hofstetter [26] and Armero and Kim [27]. In the Partial Domain Tracking Algorithm of Feist 
and Hofstetter [26], the crack-tracking problem is solved for a subset of the domain that is potentially intersected by a crack. Armero and Kim [27] propose an element local solution where the Laplace problem is solved for one front element at a time. However, the approach presented here is distinct for two reasons (a) we demonstrate that a local solution is required not just from an efficiency perspective but also to prevent spurious crack path, and (b) the local solution procedure we are advocating involves all elements just ahead of the crack front unlike either of the methods described above. Finally, while we use the Hansbo method [3] to enhance the kinematics of the elements intersected by the discontinuity, the crack-tracking approach presented here is equally applicable to both the X-FEM and E-FEM techniques.

The paper is organized as follows. In Section 2, we discuss the governing equations and the associated weak formulations. In Section 3, we briefly summarize the spatial discretization used. In Section 4, we first motivate the need for the local crack-tracking approach and then describe the crack propagation algorithm in detail. In Section 5, we illustrate the performance of the method on several benchmark problems. Finally, in Section 6 we offer some concluding remarks and an outlook for our work.

\section{Governing equations and weak formulation}

We begin by considering a domain $\Omega$ (an open subset in $\mathbb{R}^{3}$ ) and its boundary $\Gamma$ as shown in Figure 1. Further, we consider $\Gamma_{c}$ to represent a crack surface with $\Gamma_{\mathrm{c}}^{1}$ and $\Gamma_{\mathrm{c}}^{2}$ representing the initially coincident crack faces. The governing equations for small deformation elastostatics are now given in direct notation as:

$$
\begin{array}{rll}
\boldsymbol{\nabla} \cdot \boldsymbol{\sigma}=\mathbf{0} & \text { in } & \Omega, \\
\mathbf{u}=\mathbf{u}^{\mathrm{d}} & \text { on } & \Gamma_{\mathrm{d}}, \\
\boldsymbol{\sigma} \cdot \mathbf{n}=\mathbf{h} & \text { on } & \Gamma_{\mathrm{n}}, \\
\boldsymbol{\sigma} \cdot \mathbf{n}=\mathbf{0} & \text { on } & \Gamma_{\mathrm{c}}^{1}, \\
\boldsymbol{\sigma} \cdot \mathbf{n}=\mathbf{0} & \text { on } & \Gamma_{\mathrm{c}}^{2}
\end{array}
$$

where $\boldsymbol{\sigma}$ and $\mathbf{u}$ denote the Cauchy stress and displacement fields in domain $\Omega$, respectively, and $\mathbf{n}$ denotes the unit outward normal. The displacement is fixed to the prescribed field $\mathbf{u}^{\mathrm{d}}$ on the Dirichlet portion of the boundary, and $\mathbf{h}$ denotes the prescribed traction on the Neumann portion of the boundary. The crack surfaces $\Gamma_{\mathrm{c}}^{1}$ and $\Gamma_{\mathrm{c}}^{2}$ are assumed to be traction free. Further, assuming small deformations, strain and displacement fields can be related 


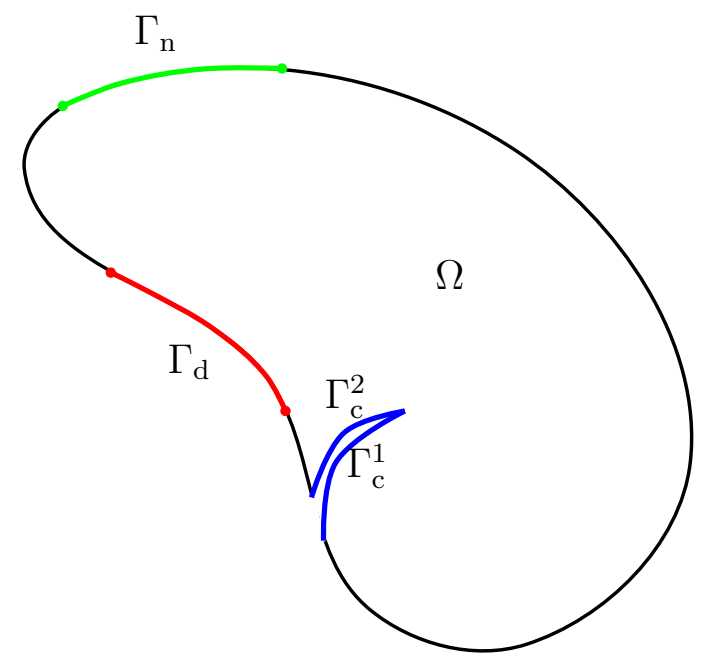

Figure 1: Notation for the model problem. Domain $\Omega$, the Dirichlet boundary $\Gamma_{\mathrm{d}}$ and the Neumann boundary $\Gamma_{\mathrm{n}}$ with prescribed non-zero tractions are as shown. $\Gamma_{\mathrm{c}}^{1}$ and $\Gamma_{\mathrm{c}}^{2}$ represent the initially coincident crack faces. The complementary part of the boundary is traction free. The normal to the boundary of a domain is considered to point outwards from the domain.

as:

$$
\varepsilon=\nabla_{s} \mathbf{u}
$$

where the symbol $\boldsymbol{\nabla}_{s}$ represents a symmetric gradient operator. Finally, we assume a linear elastic response for the constitutive relationship in the bulk domain such that:

$$
\boldsymbol{\sigma}=\mathbb{C}: \varepsilon \text { in } \Omega,
$$

where $\mathbb{C}$ denotes the fourth-order elasticity tensor. The weak form of the governing equations described above can be derived as:

Find $\mathbf{u} \in \mathcal{U}$ such that:

$$
\int_{\Omega} \nabla_{s} \mathbf{w}: \mathbb{C}: \nabla_{s} \mathbf{u} \mathrm{d} \Omega=\int_{\Gamma_{\mathrm{n}}} \mathbf{w} \cdot \mathbf{h} \mathrm{d} \Gamma \quad \forall \mathbf{w} \in \mathcal{V},
$$

where $\mathbf{w}$ represent the appropriate weight functions, $\mathcal{U}$ and $\mathcal{V}$ are spaces of sufficiently smooth functions for the displacements and variations, respectively. 


\section{Spatial discretization}

We discretize the domain $\Omega$ into a set of standard, non-overlapping hexahedral elements. We enhance the kinematics of the cracked elements by means of a Heaviside function. In particular, we follow the now standard Hansbo method (also known as the phantom-node method) to incorporate the embedded discontinuity. For the sake of completeness, a brief summary of the constructed approximation for the discontinuous displacement field and the resulting discrete matrices is provided below. The reader is referred to Hansbo and Hansbo [4, 3], Mergheim et al. [35], and Song et al. [36] for a much more detailed description.

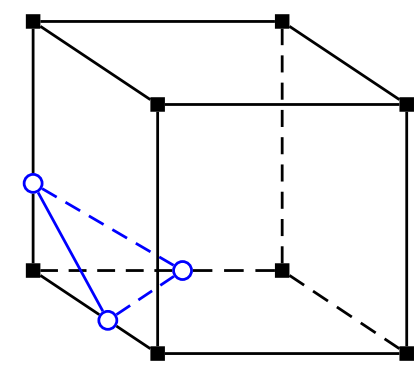

(a) An element cut by a crack surface

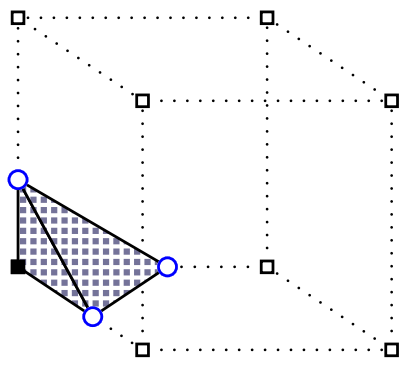

(b) Child element: $\Omega_{e}^{1}$

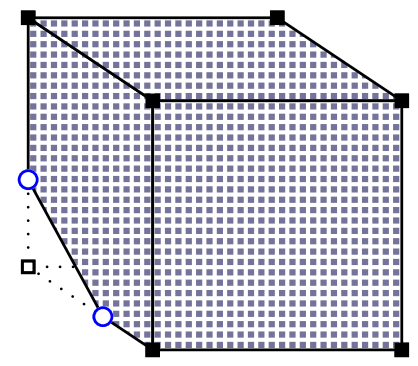

(c) Child element: $\Omega_{e}^{2}$

Figure 2: Cut elements in the Hansbo formulation. The black squares represent the nodes of the background mesh, the hollow square nodes are the phantom nodes and the blue circles are the interface vertices. For the partial elements, integration is carried over the filled region that represents the material part of the element.

For uncut elements, the displacement field within the element is continuous and is constructed in a standard manner. For the cut elements, 
displacement field is discontinuous and constructed independently on either side of the crack such that:

$$
\mathbf{u}(\mathbf{x})= \begin{cases}\mathbf{u}^{1} & \text { if } \mathbf{x} \in \Omega_{e}^{1} \\ \mathbf{u}^{2} & \text { if } \mathbf{x} \in \Omega_{e}^{2}\end{cases}
$$

where $\Omega_{e}^{1}$ and $\Omega_{e}^{2}$ denote the volume on either side of the discontinuity (see Figure 2). The local displacement approximation is constructed in terms of the nodal displacements and functions with compact support such that:

$$
\left.\mathbf{u}^{\mathrm{m}}(\mathbf{x})\right|_{\boldsymbol{\Omega}_{\mathbf{e}}}=\sum_{i=1}^{i=n_{e n}} H^{\mathrm{m}} N_{i}^{\mathrm{m}} \mathbf{u}_{\mathbf{i}} \quad \mathrm{m}=1,2,
$$

where $n_{e n}$ represents the number of nodes per finite element. The Heaviside function determines if the shape functions are active on one or the other side of a discontinuity:

$$
H^{\mathrm{m}}(\mathbf{x})= \begin{cases}1 & \text { if } \mathbf{x} \in \Omega_{e}^{\mathrm{m}}, \\ 0 & \text { otherwise. }\end{cases}
$$

In practice, the above formulation is identical to replacing each cut element with two partially integrated elements (see Figure 2 for a representative case. Partial elements can be constructed in a similar manner for all other possible configurations of cuts.)

On introducing the above discretization into the variational formulation (4), we get the discrete statement of equilibrium:

$$
\mathbf{K u}=\mathbf{F} \text {, }
$$

where $\mathbf{K}$ represents the global stiffness matrix and $\mathbf{u}$ denotes the vector of nodal displacements. The right hand side vector of external forces $\mathbf{F}$ is assembled from element contributions to the traction boundary conditions on the Neumann boundary:

$$
\mathbf{F}=\underset{e \in \mathcal{E}_{\mathrm{uc}}}{\mathbf{A}} \int_{\Gamma_{\mathrm{n} e}} \mathbf{N}^{\mathrm{T}} \mathbf{h} \mathrm{d} \Gamma_{e}+\sum_{\mathrm{m}=1}^{2} \underset{e \in \mathcal{E}_{\mathrm{cut}}}{\mathbf{A}} \int_{\Gamma_{\mathrm{n} e}^{\mathrm{m}}} \mathbf{N}^{\mathrm{T}} \mathbf{h} \mathrm{d} \Gamma_{e},
$$

where $\mathcal{E}_{\text {uc }}$ denotes the set of uncut elements and $\mathcal{E}_{\text {cut }}$ denotes the set of cut elements. The shape function matrix is denoted by $\mathbf{N}$, and $\mathbf{h}$ denotes the vector of applied tractions, and $\mathbf{A}$ denotes the assembly operator. For 
cut elements, integration is only performed on the material portion of the Neumann boundary: $\Gamma_{\mathrm{n} e}^{\mathrm{m}}=\Gamma_{\mathrm{n} e} \cap \Omega_{e}^{\mathrm{m}}$. Finally, the stiffness matrix $\mathbf{K}$ is assembled as follows:

$$
\mathbf{K}=\underset{e \in \mathcal{E}_{\mathrm{uc}}}{\mathbf{A}} \int_{\Gamma_{\mathrm{n} e}} \mathbf{B}^{\mathrm{T}} \mathbf{D B} \mathrm{d} \Omega_{e}+\sum_{\mathrm{m}=1}^{2} \underset{e \in \mathcal{E}_{\mathrm{cut}}}{\mathbf{A}} \int_{\Omega_{e}^{\mathrm{m}}} \mathbf{B}^{\mathrm{T}} \mathbf{D B} \mathrm{d} \Omega_{e},
$$

where the matrix of shape function derivatives is denoted by $\mathbf{B}$ and $\mathbf{D}$ represents the elasticity tensor. Integration of the weak form for the created children is only performed on the material side of the element. At the expense of accuracy, for partial elements, the calculated stiffness matrix is simply scaled by the volume fraction on the material side of the element. Strictly speaking, this strategy is only correct for constant strain triangular and tetrahedral elements. Several strategies exist for ensuring the discontinuous functions are accurately integrated $[37,1,38,39,40]$ but for the purpose of this study the volume fraction strategy proved sufficient.

\section{Crack propagation}

In embedded methods, a complete description of a crack propagation algorithm requires two conditions to be specified for every candidate element. The first one evaluates whether the element has failed while the second one determines the intersection points between the crack surface and the newly failed element.

\subsection{Failure criterion}

We follow a maximum principal stress based criterion to advance the crack. Consider a spectral decomposition of the Cauchy stress tensor:

$$
\boldsymbol{\sigma}=\sum_{a=1}^{a=3} \lambda_{a} \boldsymbol{n}_{\boldsymbol{a}} \otimes \boldsymbol{n}_{\boldsymbol{a}}
$$

Accordingly, a candidate element is considered to have failed if the following condition holds:

$$
\lambda_{\max }-\sigma_{f}>0,
$$

where $\lambda_{\max }=\max \left(\lambda_{1}, \lambda_{2}, \lambda_{3}\right)$ represents the maximum eigenvalue from a spectral decomposition of the Cauchy stress tensor $\boldsymbol{\sigma}$ and $\sigma_{f}$ is a material parameter representing the tensile strength of the material. 
It is noted that the formulation presented in equation (1) does not include cohesive tractions on the surface of the crack. Typically the use of a stress criteria, such as the one proposed in equation (12), without the inclusion of a mechanism to remove the stress singularity (e.g. cohesive tractions) results in a method that will exhibit a strong mesh dependence associated with the singularity. In other words, for any arbitrary loading, the solution will always exceed the stress criteria under mesh refinement. However, in this work we are focused solely on the issues associated with determining the path of the fracture once the propagation criteria has been reached rather than the physical applicability of the propagation criteria.

It is also noteworthy that we assume that the crack propagates an entire element at a time $i$. e . crack does not terminate within an element so that the crack front always lies along boundary of the underlying finite element mesh. Together with the above assumption of a lack of a fracture process zone, this simplification could result in some mesh-dependence of results especially for fatigue and dynamic fracture applications. However, this limitation can be addressed through the addition of an appropriate traction-separation law at the crack surface.

\subsection{Crack location}

In many explicit crack-tracking approaches, the crack surface is assumed to be planar within a given element. Under this assumption, in order to describe the crack plane and its intersections with the underlying volume element, we need to define a crack normal and a given point on the crack. The maximum principal stress direction given by the eigenvector $\mathbf{n}$ associated with $\lambda_{\max }$ serves as the crack normal. Assuming the crack is continuous as it propagates, previously fractured neighboring elements provide a spatial point that lies on the crack. This is a viable strategy in 2D and yields a unique definition of the crack surface. However, in 3D, under the assumptions of a locally planar crack, this is an over-constrained problem as the number of points that lie on the crack surface depends on the number of cut neighbors. In fact, the simplest configuration with just one cut neighbor is still overconstrained as we now have to define a plane with two points and a normal. Clearly, in more general scenarios enforcing continuity as well as the correct crack normal is rather challenging and requires ad hoc solutions (see Areias and Belytschko [11] and Oliver et al. [24] for a discussion). Oliver et al. [24] presented a remarkably simple global tracking algorithm to circumvent this difficulty. In the following discussion, we first summarize the 
global crack-tracking method presented by Oliver et al. [24] in Section 4.2.1. In Section 4.2.2, we illustrate the pathological cases for the global method by means of a simple example. Finally, in Section 4.2.3, we propose an alternative solution to address the pathology.

\subsubsection{Global crack-tracking method}

In the global crack-tracking method, Oliver et al. [24] proposed solving for a scalar field, $\theta$, that tracks all potential crack trajectories in the domain. Any given crack is then tracked in 3D by an iso-surface of this scalar field $\theta$. To lend concreteness to the formulation, let us consider a crack with a given normal $\mathbf{n}$. Recalling that $\theta=$ constant on a crack surface, from the definition of a directional derivative:

$$
(\mathbb{I}-\mathbf{n} \otimes \mathbf{n}) \cdot \nabla \theta=\mathbf{0}
$$

The above equation represents a flux like quantity and enables us to recast the equation in terms of a classical Laplace equation with anisotropic material constant $\mathbb{K}=\mathbb{I}-\mathbf{n} \otimes \mathbf{n}$, so that:

$$
\begin{aligned}
\mathbf{q}=\mathbb{K} \cdot \nabla \theta=\mathbf{0} & \text { in } \Omega, \\
\nabla \cdot \mathbf{q}=\mathbf{0} & \text { in } \Omega,
\end{aligned}
$$

and the natural boundary condition (again by construction)

$$
\mathbf{q} \cdot \mathbf{m}=\mathbf{0} \quad \text { on } \partial \Omega
$$

where $\mathbf{m}$ represents the outward pointing normal to the boundary of the domain. The above equation can then be solved on the same mesh as the mechanical problem in a straightforward manner. This problem is always solved on the initial background mesh, i.e., the cut elements are not replaced by their superposed counterparts from the Hansbo formulation. It was also suggested that to remove any numerical artifacts arising from the singular nature of the "conductivity" tensor $\mathbb{K}$, a potentially "small" perturbation value could be added to offset it such that we use $\mathbb{K}=(1+\varepsilon) \mathbb{I}-\mathbf{n} \otimes \mathbf{n}$ in computations instead. Notice that the above definition of the boundary value problem (equations (15) and (16)) is ill-posed unless Dirichlet data are specified. In order to get a non-trivial solution, $\theta$ values at two nodes that do not lie on the same iso-surface are arbitrarily specified. Since the equation (15) is a classical field equation that is commonly used to solve 
$\overline{\text { Algorithm } 1 \text { Algorithm summarizing the steps involved in modeling crack }}$ propagation with the global crack-tracking approach

Begin initialize

Modify mesh topology:

Generate overlapping elements for all elements intersected by

a pre-specified fracture

Generate a list of elements that lie at the crack front: $\mathcal{E}_{\text {front }}$

Thermal solve for $\theta$ :

Get normals in pre-fractured elements from the crack plane

Get conductivity tensor:

For pre-fractured elements: $\mathbb{K}=(1+\varepsilon) \mathbb{I}-\mathbf{n} \otimes \mathbf{n}$

For all other elements: $\mathbb{K}=\varepsilon \mathbb{I}$

Apply Dirichlet boundary conditions at arbitrarily chosen loca-

tions

Get the iso-surface value to track the crack

End initialize

Set fracturesResolved $=$ false

while fracturesResolved $=$ false do

Mechanical solve

Check for failed elements in $\mathcal{E}_{\text {front }}$ :

Store all failed elements in a list of elements that have failed at $i^{\text {th }}$ iteration $\mathcal{E}_{\text {cut }}^{i}$

If $\mathcal{E}_{\text {cut }}^{i}$ is empty, set fracturesResolved $=$ true

if fracturesResolved is false then

Thermal solve for $\theta$ :

Get conductivity tensor for every element: $\mathbb{K}=(1+\varepsilon) \mathbb{I}-\mathbf{n} \otimes \mathbf{n}$

Apply Dirichlet boundary conditions for all nodes $\mathcal{N} \in \mathcal{E}_{\text {cut }}$

Modify mesh topology:

Get intersection points for all elements in $\mathcal{E}_{\text {cut }}^{i}$ based on the isosurface values for $\theta$

Generate overlapping elements for all elements in $\mathcal{E}_{\text {cut }}^{i}$ end if

Update the lists $\mathcal{E}_{\text {cut }}, \mathcal{E}_{\text {front }}$ and clear the list $\mathcal{E}_{\text {cut }}^{i}$

end while 
heat-conduction problems, we will refer to the crack-tracking equation as a thermal problem for convenience.

In the original method [24], Oliver et al. did not explicitly discuss the treatment of pre-specified embedded fractures in the method. In order to clarify our implementation of this method, we briefly discuss our approach for their inclusion here. The primary difficulty with the inclusion of embedded pre-fractures arises from the absence of the nodal field $\theta$ at the initialization stage. The nodal field $\theta$ is required at the problem initialization stage to identify the elements that are crossed by the pre-specified fracture so that they can be kinematically enhanced. One could always initialize the field as a signed-distance function to a crack. However, this methodology forces us to track every crack as $\theta=0$ which could be problematic for the case when there are multiple cracks in the domain. Instead, we explicitly split elements with the provided input of cracks as piecewise planar surfaces at the initialization step. Now, at the end of this step the nodal field $\theta$ is initialized with Dirichlet boundary conditions at arbitrarily specified points and a conductivity tensor specified as $\mathbb{K}=(1+\varepsilon) \mathbb{I}-\mathbf{n} \otimes \mathbf{n}$ in elements that are pre-fractured. The normal $\mathbf{n}$ in these elements is given by the normal to the piecewise planar crack surface. In all other elements, the conductivity tensor is specified as $\mathbb{K}=\varepsilon \mathbb{I}$. At the end of the initialization phase, for every crack, an arithmetic mean of the $\theta$ values is evaluated at the crack front to obtain the iso-surface value that tracks the crack. In other words, for a crack $C_{i}$ :

$$
\theta_{i}=\frac{1}{n_{c u t}} \sum_{n=1}^{n=n_{\text {cut }}} \theta_{n}
$$

where $n_{\text {cut }}$ represents the number of cut edges, $\theta_{n}$ denotes the value of the field at the $n^{\text {th }}$ cut edge, and $\theta_{i}$ is the iso-surface label to track the crack $C_{i}$. For every subsequent thermal solve, the nodes of all the cracked elements serve as the Dirichlet boundaries and the Dirichlet boundaries used for initializing the field are no longer necessary. A summary of the staggered mechanical and thermal solution steps is given in Algorithm 1.

\subsubsection{Pathologies in the global crack-tracking method}

In the discussion above the Dirichlet boundary conditions for $\theta$ are little but a footnote. However, in practice the performance of the algorithm is quite

sensitive to them and can cause highly spurious behavior in the resulting crack path. Jäger et al. [30, 31, 32] have previously reported this numerical 
sensitivity and suggested geometry and mesh-dependent boundary conditions as a possible means to address it. This sensitivity is illustrated by means of a simple example below.

Consider a computational domain of $\Omega=(0,1) \times(0,1) \times(0,1)$ that is fixed at the boundary $y=0$ and is uniaxially stretched at the boundary $y=1$ by a prescribed displacement of $u_{y}=0.1$ units. The Young's modulus and Poisson's ratio are prescribed as $E=200$ units and $\nu=0.3$. The boundaries $z=0$ and $z=1$ are fixed in the z-direction so that we have plane-strain conditions. We use a structured cartesian grid with 5 divisions in $\mathrm{x}$ and $\mathrm{y}$ and 1 division in z. A crack is initialized at $y=y_{*}$ and extends to $x=0.2$. We choose $y_{*}=0.5$ and $y_{*}=0.59$ for the two example cases and illustrate the performance. For both examples, at the initialization stage, the boundaries $y=0$ and $y=1$ are treated as Dirichlet boundaries and $\theta$ is specified as -1 and 1 respectively on these boundaries. For subsequent solves, the value of $\theta$ is retained for the nodes of cut elements and only these nodes then serve as Dirichlet conditions.

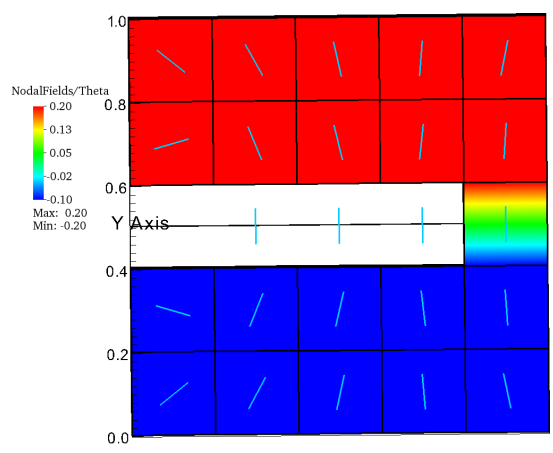

(a) Crack initialized at $y_{*}=0.5$

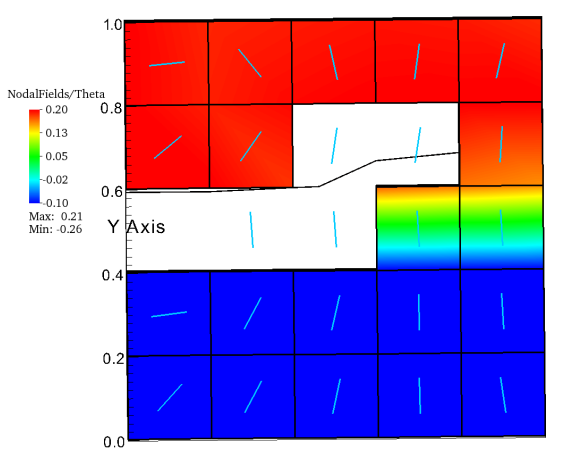

(b) Crack initialized at $y_{*}=0.59$

Figure 3: A pseudocolor plot of the scalar field $\theta$, principal directions shown as cell-centred blue lines and the resulting crack path for the two example cases: crack is initialized at $y_{*}=0.5$ (on left) and $y_{*}=0.59$ (on right).

The resulting crack path for both these cases just before the crack completely separates the domain into two disjoint sets is shown in Figure 3. For a clearer understanding, we have also plotted the maximum principal stress directions in the elements intersected by the crack, shown as cell-centred blue lines in Figure 3. Notice that while for the first case when $y_{*}=0.5$, the crack path looks to conform well to the crack normals (Figure 3(a)), there is an unnatural kink appearing in the second case when the crack first crosses the 
surface $y=0.6$ (Figure 3(b)). In the first element that appears at this transition, the crack surface and the directions of the maximum principal stress do not seem to agree.

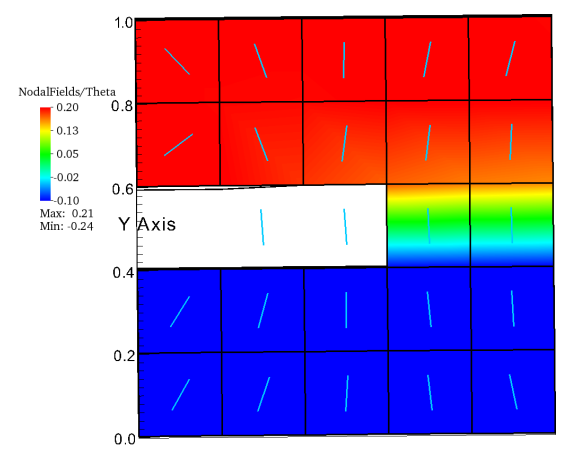

Figure 4: A pseudocolor plot of the scalar field $\theta$ just before the crack first crosses the surface $y=0.6$. Crack is initialized at $y_{*}=0.59$. It can be noticed that the gradient of the scalar field in the element at the crack front is small.

In order to understand this discrepancy better, we focus on the step immediately before this transition occurs in Figure 4. We now notice that the observed pathology appears as the crack surface first enters a region where $\theta \approx$ constant. In such regions, the concept of an iso-surface becomes ill-defined and spurious behavior is more likely. In order for the equations to serve their intended purpose to track the crack, it is imperative that $\nabla \theta$ in the direction perpendicular to the crack surface in the crack-tip elements is sufficiently large. We also contrast this with the first case where the crack path behaves in an expected manner and notice that in this case the gradient in the crack-front elements is always well-defined and the crack never enters a region with $\theta \approx$ constant.

In our opinion, this is the primary reason for the algorithm's sensitivity to the Dirichlet boundary conditions used for the thermal problem. If we solve the problem again by treating the boundaries $y=0$ and $y=1$ also as Dirichlet boundaries in addition to the nodes of the cut elements, the algorithm performs better because a strong gradient is always ensured in the elements at the crack front (see Figure 5). The challenge then lies in identifying the right boundary conditions that result in a robust algorithm. While this has been addressed by Jäger et al. [32] to some extent where they prescribe mesh-based and geometry based boundary conditions, we noticed that spurious behavior could still result if the boundary conditions do not 


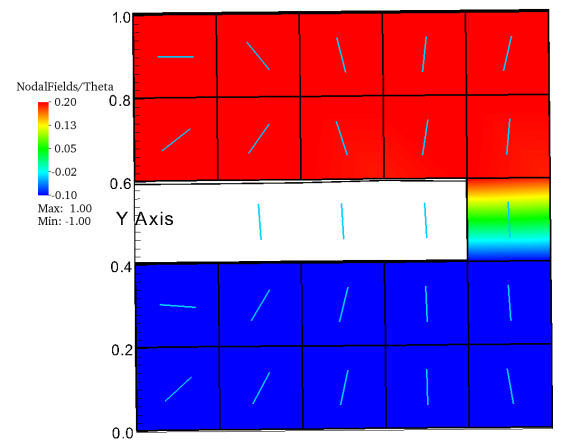

Figure 5: A pseudocolor plot of the scalar field $\theta$ with a crack initialized at $y_{*}=0.59$. The thermal boundary conditions are specified at all the nodes of cracked elements as well as at boundaries $y=0$ and $y=1$. A strong gradient is maintained in the elements at the front and resolves the pathology.

ensure a strong gradient in the elements at the crack front (see Section 5 for an illustration).

\subsubsection{Local crack tracking algorithm}

We propose a local crack-tracking algorithm here to address the above described sensitivity. We propose restricting the solution of the anisotropic Laplace equation to a local region extending to the elements just ahead of the crack front. The nodes that belong to the cut faces from elements cut in the previous step naturally serve as Dirichlet boundary conditions. This also ensures that in the elements at the crack front, a strong gradient is always maintained. In other words, the crack-tracking equation can be stated as follows:

Find $\theta$ such that:

$$
\begin{aligned}
\mathbf{q}=\mathbb{K} \cdot \nabla \theta=\mathbf{0} & \text { in } \mathcal{E}_{\text {front }}, \\
\nabla \cdot \mathbf{q}=\mathbf{0} & \text { in } \mathcal{E}_{\text {front }},
\end{aligned}
$$

and the boundary conditions:

$$
\begin{aligned}
\theta & =\theta_{n} \quad \text { on } \partial \mathcal{E}_{\theta}=\mathcal{E}_{\text {front }} \cap \mathcal{E}_{\text {cut }}, \\
\mathbf{q} \cdot \mathbf{m} & =\mathbf{0} \quad \text { on } \partial \mathcal{E}_{\text {front }} \backslash \partial \mathcal{E}_{\theta},
\end{aligned}
$$

where $\mathbb{K}=(1+\varepsilon) \mathbb{I}-\mathbf{n} \otimes \mathbf{n}$ is the anisotropic conductivity tensor, $\mathcal{E}_{\text {front }}$ denotes the set of elements at the crack front, $\mathcal{E}_{\text {cut }}$ denotes the set of cut 

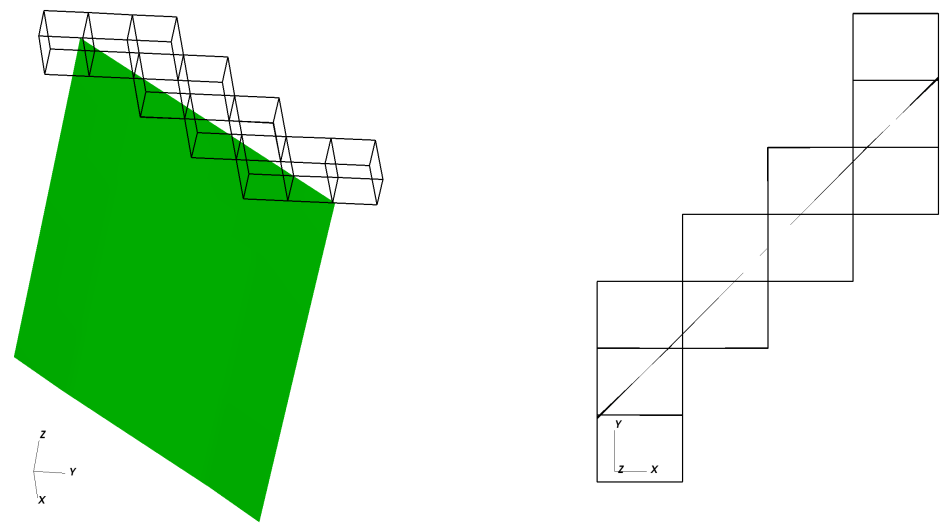

Figure 6: An illustration of the computational domain $\mathcal{E}_{\text {front }}$ used in the solution of the thermal problem in the proposed approach. The top-view of the domain is shown on right and a three-dimensional representation is shown on the left. In these figures the elements of $\mathcal{E}_{\text {front }}$ are shown as wireframe illustrations, while the crack surface is shown in green. Note that $\mathcal{E}_{\text {front }}$ contains all elements that have a face cut by the crack surface, but are not already cut themselves.

elements and $\theta_{n}$ are the nodal solutions for the thermal field calculated at the previous iteration. Figure 6 shows an illustration of the domain $\mathcal{E}_{\text {front }}$ used for the local solution of the thermal problem.

The maximum principal stress direction, $\mathbf{n}$, is calculated from a "nonlocal stress tensor" that is a weighted average of the stress from the elements in the neighborhood of the crack-tip. We follow the procedure discussed in Wells and Sluys [41] to obtain the weighted stress $\boldsymbol{\sigma}$, such that:

$$
\boldsymbol{\sigma}=\sum_{e=1}^{e=n_{e l e}} w_{e} \boldsymbol{\sigma}_{e} V_{e}
$$

where $w_{e}$ is an element weighting factor, $V_{e}$ is the element volume, and $n_{\text {ele }}$ denotes the number of elements. The stress in each element $\boldsymbol{\sigma}_{e}$ is given by an arithmetic mean of the stresses from every gauss point. The weight functions are calculated as:

$$
\hat{w}(r)=\frac{1}{2 \sqrt{\pi}} \exp \left(\frac{-r^{2}}{2 l^{2}}\right), \quad w(r)=\frac{\hat{w}(r)}{\sum_{e=1}^{n_{\text {ele }}} \hat{w}_{e} V_{e}},
$$

where $r$ is the distance of the element center to the center of the element at the crack front and $l$ is the decay length that determines the smoothing radius for the non-local averaging. 


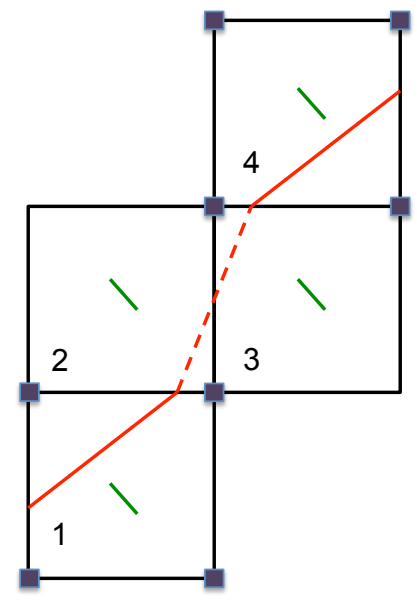

(a) Predicted crack path if only elements cut in the current iteration are included in the domain for the thermal problem.

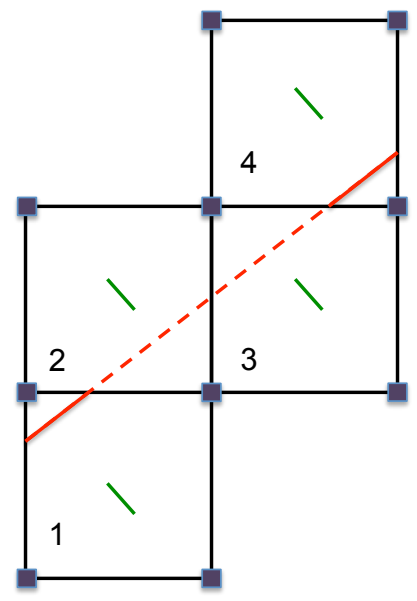

(b) Predicted crack path if all elements in the crack front are included in the domain for the thermal problem.

Figure 7: An illustration of the pathology that results if the thermal problem is not solved on a domain that includes all elements in the crack front. The red lines represent a top view of the crack front. The solid lines represent the consolidated crack path and the dashed lines represent the allowed crack path in adjacent elements. The solid squares represent the nodal locations that have been solved for the scalar field $\theta$. 
As a quick remark, we point out that solving the thermal equations in all the elements ahead of the crack front regardless of whether they get split in the current iteration ensures that the crack path remains smooth as it propagates. If one chooses to solve the equations in only those elements that are split in the current iteration, a case could arise where two completely disconnected elements are split in such a way that they predetermine the crack surface for the elements connecting them. In this scenario, while the crack surface in each of these elements will independently conform to the direction of the maximum principal stress in these elements, the global surface would be erroneous (see Figure 7 for an explanation). Consider a top view of the crack front as shown in Figure 7(a). The solid red lines represent the top view of the crack in failed elements while the dashed lines represent the potential path for a crack surface in the intact front elements. The green lines represent the direction of the maximum principal stress. Now, consider a scenario in which only elements 1 and 4 have failed in one step. If the thermal solve is only conducted in these elements, it may result in a scenario where the failure surface in elements 2 and 3 is predetermined in a manner inconsistent with the intended direction of propagation. On the other hand, if the solution is conducted for all four elements at the same time, the resulting solution tries to conform with the direction in all the elements as shown in Figure 7(b)

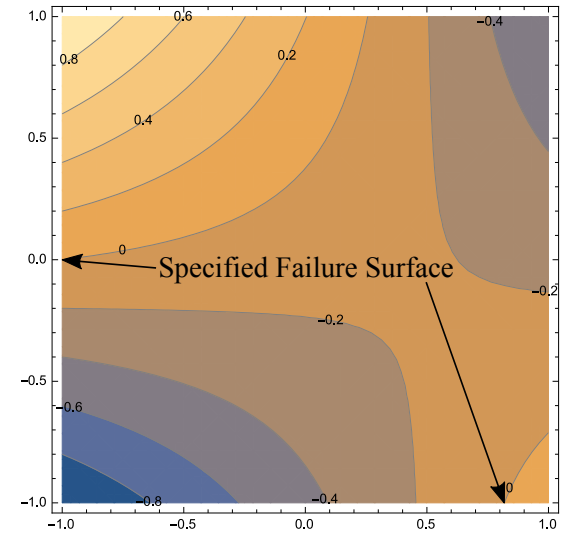

(a) Gaussian Quadrature

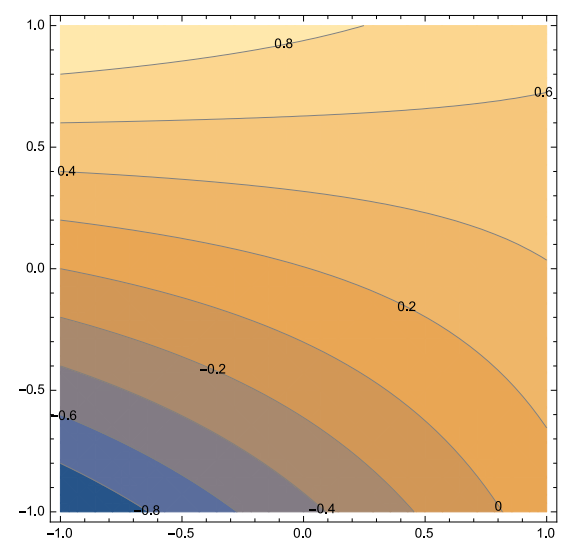

(b) Nodal Quadrature

Figure 8: A contour plot of the solution for the scalar field $\theta$ on a single quadrilateral element with Gaussian Quadrature (on left) and Nodal Quadrature (on right). The failure surface is tracked by the zero isoline in the above plot. 
Finally, we utilize a nodal integration procedure to evaluate the local matrices for the thermal problem. The motivation for the use of nodal integration is to avoid non-unique failure surfaces that could arise in an element with bilinear or trilinear shape functions [28]. A simple example of nonunique failure surfaces may be displayed on a bilinear quadrilateral. Consider a square quadrilateral element that ranges from $(-1,1)$, and has Dirichlet boundary conditions on three of the nodes $(\theta=-1$ at $(-1,-1), \theta=0.1$ at $(1,-1)$, and $\theta=1$ at $(-1,1))$, leaving only one unspecified degree of freedom for $\theta$ at $(1,1)$. Assuming that a failure surface is tracked by the zero iso-surface of $\theta$, these boundary conditions indicate that there is an existing failure surface that connects the left edge, and the bottom edge of the element. While we could recognize that the failure surface in this element is completely pre-determined and treat such elements distinctly, it results in "special cases" and implementation challenges. Ideally the solution at the unspecified degree of freedom should be consistent with the existing failure surface in the element. However, we have observed that Gaussian quadrature allows a non-unique result. For example, if we specify a principal stress direction of $(\pi / 10)$ with respect to the horizontal axis and apply the proposed method using Gaussian quadrature, the result is a non-unique pair of failure surfaces as shown in Figure 8(a). However, if we apply nodal quadrature, the result is a single unique failure surface as shown in Figure 8(b). While no mathematical proof is offered here, it appears that the use of nodal integration reduces the cross-contributions from the diagonal nodes in the resulting discrete system, preserving the local monotonicity of the field. Although there may be cases where the nodal integration technique is insufficient, the method proved sufficient for all the numerical tests we conducted. A more sophisticated approach such as the marching cubes algorithm of Linder and Zhang [28] can be adapted to address this issue in a more rigorous way.

Algorithm 2 summarizes the approach and provides the implementational details. The example presented in Section 4.2.2 is solved again with the proposed approach and the results are presented in Figure 9. Clearly, the results do not exhibit any non-physical surface kinks regardless of the initial crack position in the element. A more thorough validation of the algorithm is conducted in the next section. To conclude this section, we remark that the proposed local approach tries to combine the advantages of the explicit crack-tracking method and Oliver's global crack-tracking approach. While the primary goal of the proposed approach is to address pathologies and sensitivity of the original algorithm to the thermal boundary conditions, we 


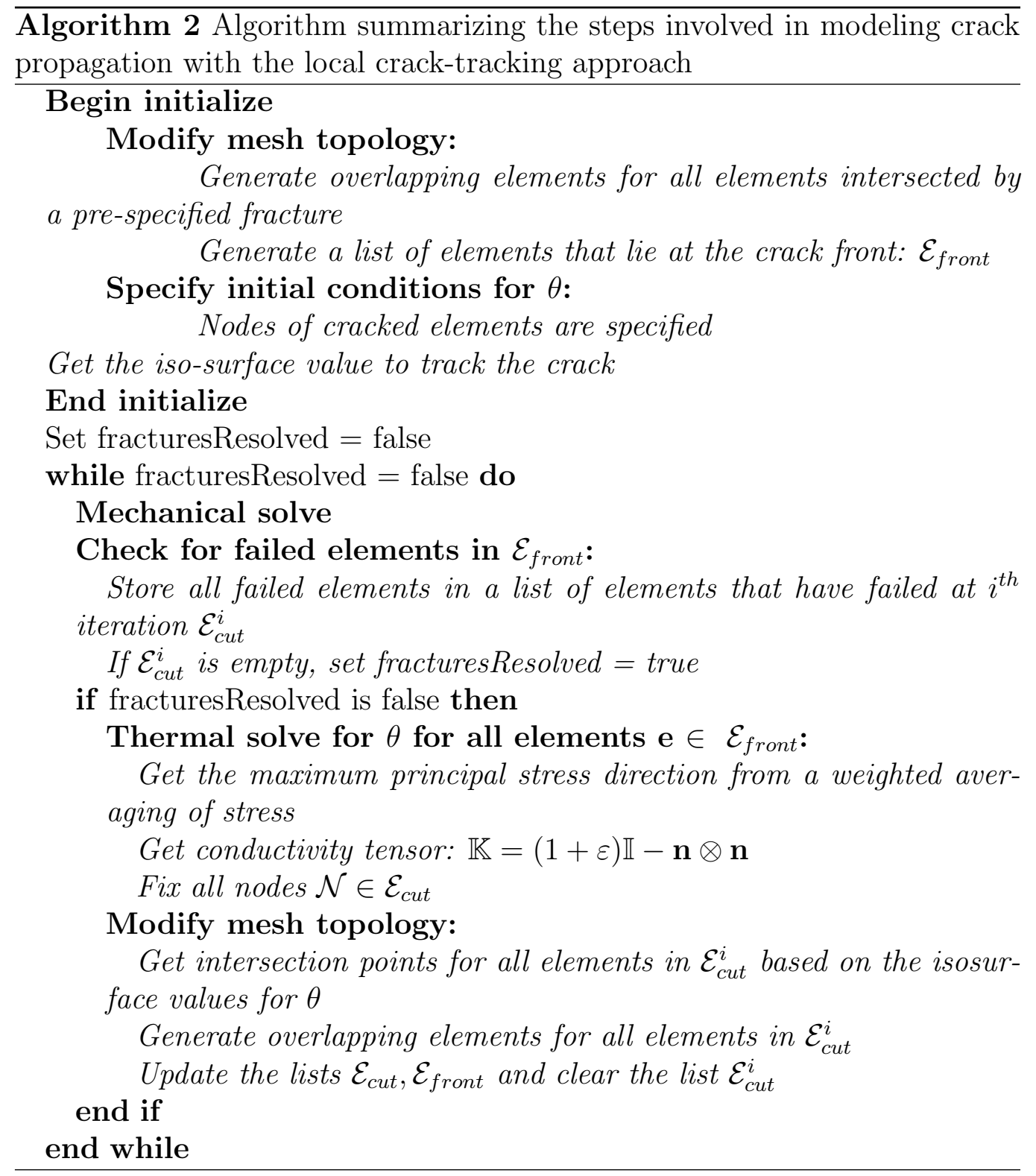




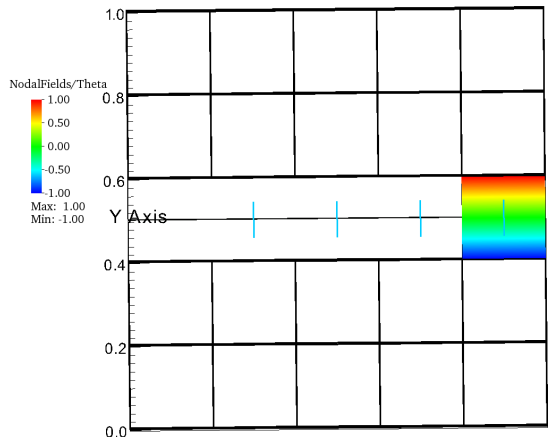

(a) Crack initialized at $y_{*}=0.5$

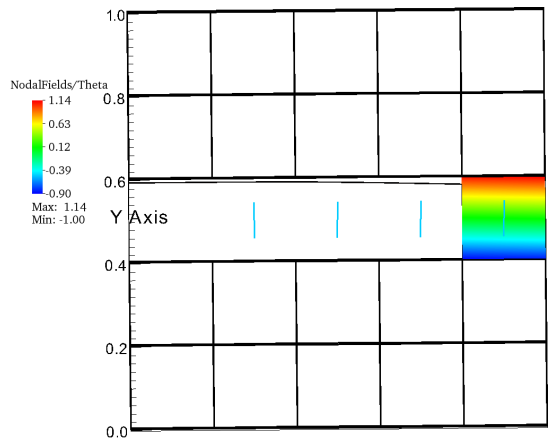

(b) Crack initialized at $y_{*}=0.59$

Figure 9: A pseudocolor plot of the thermal field $\theta$ in the element at the crack front and the resulting crack path for the cases when crack is initialized at $y_{*}=0.5$ (on left) and $y_{*}=0.59$ (on right).

also remark that a more local procedure provides an added computational benefit.

\section{Numerical examples}

In this section, we perform several numerical experiments to validate our approach. We compare our results with available literature. We also compare the results obtained from the proposed algorithm with the global algorithm of Oliver et al. [24], where necessary, to highlight its advantages. For all our numerical studies, we consider a linear-elastic constitutive behavior for the bulk material. The crack is propagated based on a maximum principal stress based criteria. Unless otherwise specified, the smoothing radius for the non-local stress averaging discussed in Section 4 is chosen as 2 elements. When using the global approach, the perturbation value $\varepsilon$ used to offset the conductivity tensor is chosen as $1 \times 10^{-4}$. For the local approach, we use $\varepsilon=1 \times 10^{-8}$. The algorithm has been implemented in GEOS: a flexible multi-scale, multi-physics simulation environment developed at Lawrence Livermore National Laboratory [42, 43, 44].

\subsection{Interacting cracks}

The interacting cracks example is a standard benchmark used to investigate the performance of the method in simulating curved fractures. The problem has been extensively studied both experimentally and numerically 


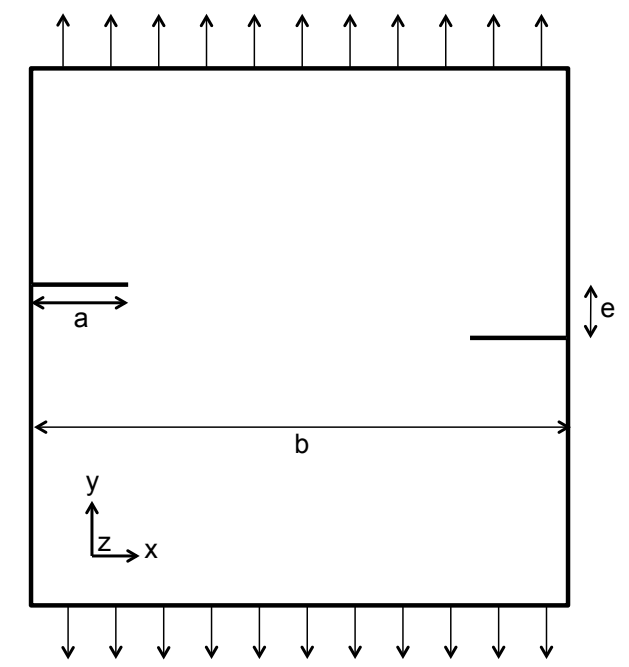

Figure 10: Illustration of the geometry and boundary conditions for the interacting cracks example.

$[45,46,47,48,49,50]$. The problem set-up is shown in Figure 10. The geometric parameters used to describe the offset between the two prexisting notched cracks determine the curvature of the crack path.

For our study, we consider the ratios $\mathrm{a} / \mathrm{b}=0.2, \mathrm{e} / \mathrm{b}=0.0595$, with $\mathrm{b}=40$ $\mathrm{mm}, \mathrm{E}=200 \mathrm{GPa}$ and $\nu=0.3$ and a failure stress $\sigma_{f}=15 \mathrm{MPa}$. We use a structured mesh of trilinear hexahedral elements with 100 divisions along the $\mathrm{x}$ and $\mathrm{y}$ directions and 4 divisions along the $\mathrm{z}$ direction. As discussed in Section 4.2.2, the performance of the algorithm is of interest when the cracks are close to an element boundary. Therefore, the initial notches are intentionally positioned at $y=18.81$ and $y=21.19$ such that the cut lies at $2.5 \%$ of the edge length. The out of plane thickness (i.e. along z-axis) is considered as $4 \mathrm{~mm}$. The problem is laterally constrained in the z-direction to replicate plane strain conditions.

The loading is applied incrementally as a kinematic boundary condition on the boundaries $y=y_{\min }$ and $y=y_{\max }$ such that the boundaries are displaced by $u_{y}=-20 \mu \mathrm{m}$ and $u_{y}=20 \mu \mathrm{m}$ respectively in each step. We initialize the $\theta$ values for all the nodes of the element that are cut by a crack such that all the nodes that lie below a crack surface are assigned a value of $\theta=-1$ while the ones above are assigned $\theta=1$. The cracks are then tracked by the iso-surface values $\theta=0.95$ and $\theta=-0.95$ respectively. 


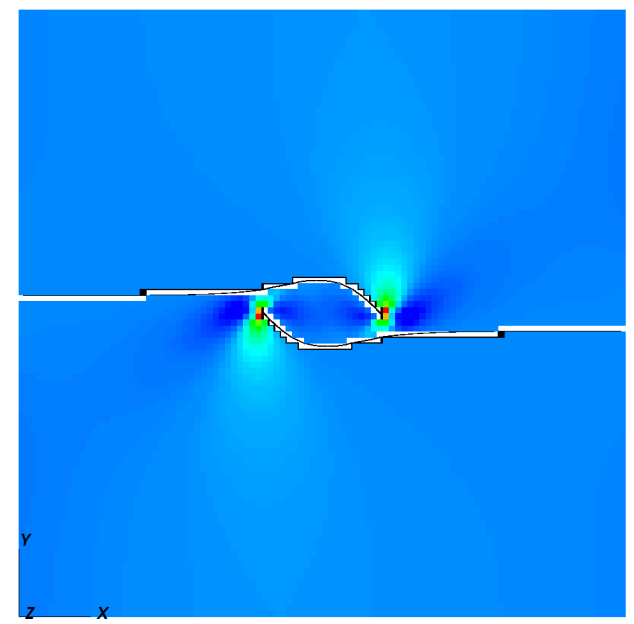

(a) Predicted crack path from the proposed local crack-tracking approach for the interacting cracks example

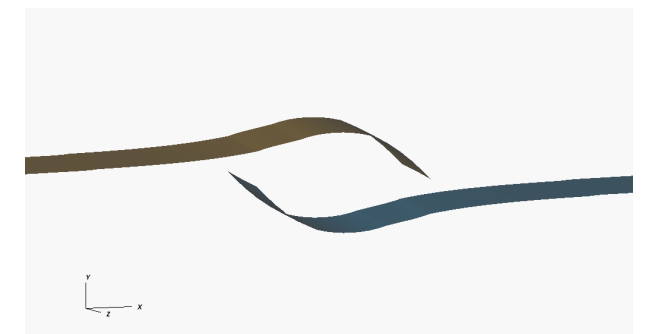

(b) A zoomed in view of the failure surface

Figure 11: A pseudocolor plot of the stress $\sigma_{y y}$ and a zoomed in view of the resulting failure surface in $3 \mathrm{D}$ 
The results obtained from our simulation at load step 50 are shown in Figure 11. In Figure 11(a), a pseudocolor plot of the stress component $\sigma_{y y}$ and a planar view of the predicted crack path is plotted. In Figure 11(b), we show the resulting failure surfaces in $3 \mathrm{D}$. As they propagate, the cracks initially tend to avoid each other and curve away from one another before turning toward one another. Our results show a good qualitative match with existing studies $[46,47]$.

Of particular interest here is the comparison of our results with the global approach described in Oliver et al. [24]. For the global approach, we initialize the signed-distance field $\theta$ using the procedure described in Section 4.2 (also see Algorithm 1). During the initialization step, the Dirichlet boundary conditions are specified on the following edges: $\mathcal{S}^{1}, \mathcal{S}^{2}, \mathcal{S}^{3}, \mathcal{S}^{4}$ such that:

$$
\begin{aligned}
& \mathcal{S}^{1}=\{\mathbf{x}: x=0, y=0, \text { and } 0 \leq z \leq 4\} \\
& \mathcal{S}^{2}=\{\mathbf{x}: x=40, y=0, \text { and } 0 \leq z \leq 4\} \\
& \mathcal{S}^{3}=\{\mathbf{x}: x=0, y=40, \text { and } 0 \leq z \leq 4\} \\
& \mathcal{S}^{4}=\{\mathbf{x}: x=40, y=40, \text { and } 0 \leq z \leq 4\}
\end{aligned}
$$

On the edges $\mathcal{S}^{1}$ and $\mathcal{S}^{2}$ we specify $\theta=-1$ while on $\mathcal{S}^{3}$ and $\mathcal{S}^{4}$ we specify $\theta=$ 1. We report results for the cases when only the cut element nodes serve as the Dirichlet boundary conditions for the thermal problem (Figure 12(a)) as well as for the case when the initially specified boundary conditions continue to be enforced in addition to the cut element nodes (Figure 12(b)).

The algorithm seems particularly sensitive to the relative position of the crack surface to the finite element mesh. For the case when the two cracks are located at $y=18.81$ and $y=21.19$ respectively, the cracks turn back on themselves as shown in Figures 12(a) and 12(b). When the cracks are moved to the middle of the element such that the two cracks are located at $y=19$ and $y=21$, the performance of the algorithm is slightly better. For this case, the cracks initially follow the expected path although the failure surface seems a little jagged as opposed to that in Figure 11(b). We notice spurious behavior from the algorithm as the fracture tips get close to one another. In particular, zooming in on the elements at the crack front (see Figure 13), as discussed in Section 4 we observe again that the performance of the algorithm is strongly dependent on $\nabla \theta$ in the elements at the crack front. Notice that the proposed approach ensures that the elements immediately ahead of the crack front have a strong gradient while in the global approach the field approaches a constant value that results in the spurious behavior. 

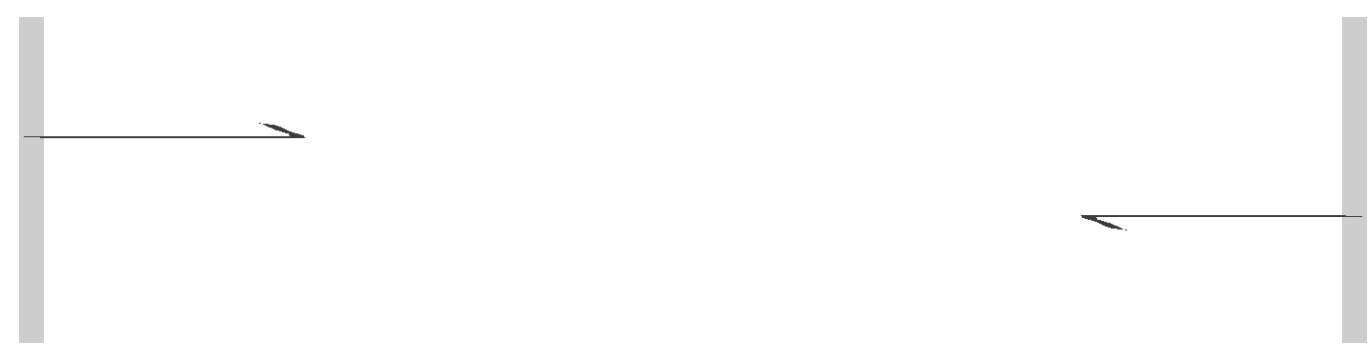

(a) Predicted crack path from the global algorithm with cracks initiated at $y=18.81$ and $y=21.19$ and thermal boundary conditions applied only on the nodes of cut elements
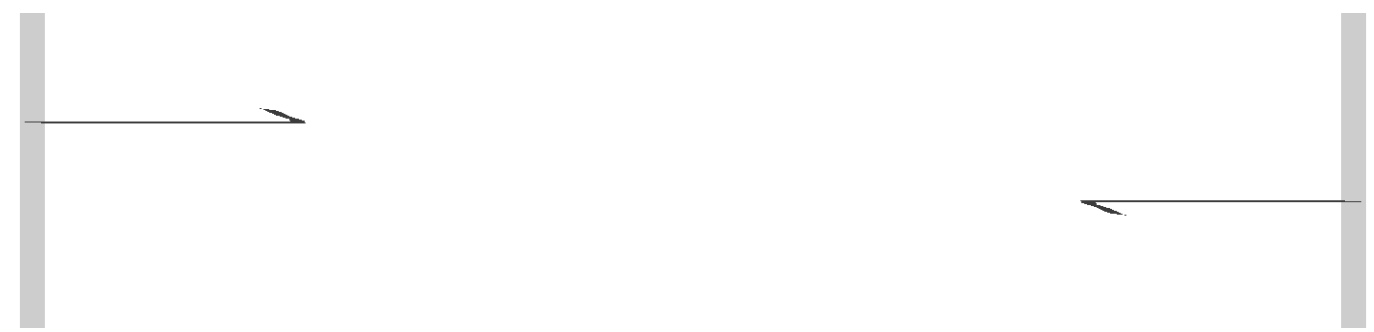

(b) Predicted crack path from the global algorithm with cracks initiated at $y=18.81$ and $y=21.19$. Thermal boundary conditions are applied on the nodes of cut elements and on the edges: $\mathcal{S}^{1}, \mathcal{S}^{2}, \mathcal{S}^{3}$, and $\mathcal{S}^{4}$

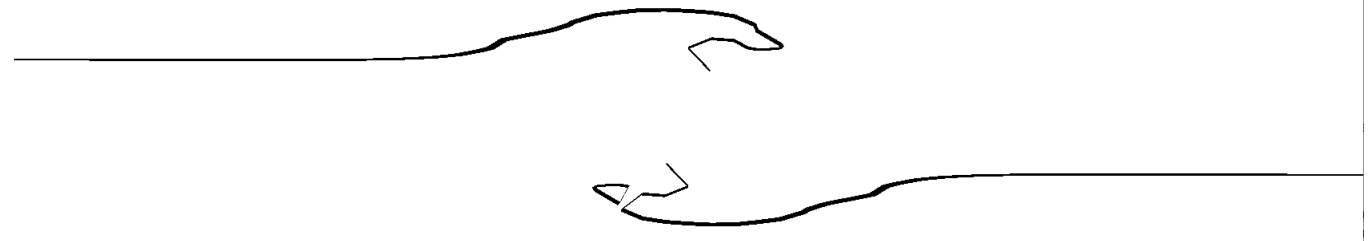

(c) Predicted crack path from the global algorithm with cracks initiated at $y=19$ and $y=21$. Thermal boundary conditions are applied only on the nodes of the cut elements

Figure 12: Predicted crack path from the global algorithm for three different cases: (a) Cracks initialized at $y=18.81, y=21.19$ and boundary conditions applied only on the nodes of cut elements, (b) Cracks initialized at $y=18.81, y=21.19$ and boundary conditions applied on the nodes of cut elements as well as at initially specified locations, and (c) Cracks initialized at $y=19, y=21$ and boundary conditions applied only on the nodes of cut elements 


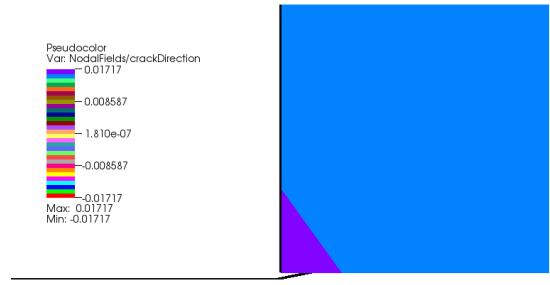

(a) Gradient in the thermal field using the global approach of Oliver et al. $[24]$

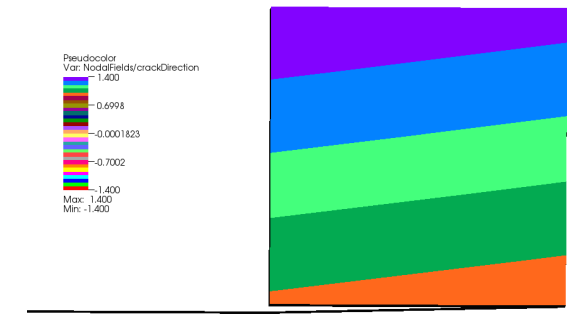

(b) Gradient in the thermal field using the proposed local approach

Figure 13: Comparison of the gradient in the thermal field in the elements ahead of the crack front using the global and proposed approaches

\subsection{Notched beam with holes}

We now consider a notched three-point bending specimen with holes as shown in Figure 14. This example was first investigated experimentally by Ingraffea and Grigoriu [51]. They reported a high sensitivity in the crack path to small changes in the initial notch position. Given this sensitivity, this example has been extensively used as a benchmark for numerical models to test the predictive capabilities of the method [52, 53, 54, 55]. Consistent with the experiments, we assign a Young's modulus of $E=3.102 \mathrm{GPa}$ and a Poisson's ratio of $\nu=0.35$. The failure stress of the material is assigned as $\sigma_{f}=7 \mathrm{MPa}$. The thickness in the z-direction is considered as $2 \mathrm{~mm}$. The mesh used for the computations is generated using CUBIT [56] and consists of 13000 unstructured hexahedral elements ( see Figure 14(b)).

We constrain the model laterally in the z-direction and vary the notch position for the three cases studied in the experiment by Ingraffea and Grigoriu [51] viz. (a) case $1: \mathrm{a}=6.0 \mathrm{~mm}$, and $\mathrm{b}=1.0 \mathrm{~mm}$, (b) case $2: \mathrm{a}=5.0$ $\mathrm{mm}$, and $\mathrm{b}=1.5 \mathrm{~mm}$, and finally (c) case $3: \mathrm{a}=6.0 \mathrm{~mm}$, and $\mathrm{b}=2.5 \mathrm{~mm}$. We report our results in the Figure 15. The results from the experiment are also plotted as a means to compare. Clearly, a good qualitative match is obtained between the numerical results from the proposed local approach and the experimental results with the crack approaching the right hole each time. 


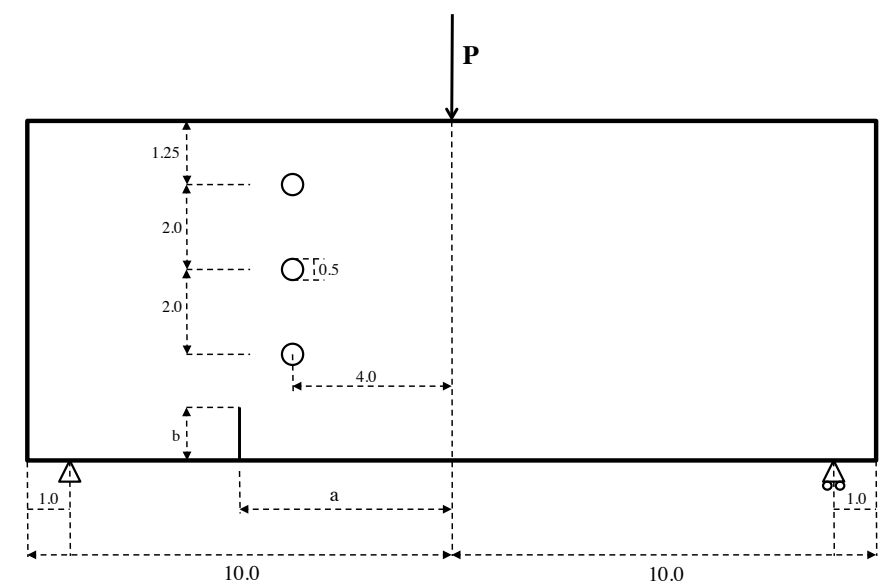

(a) Geometry and loading conditions

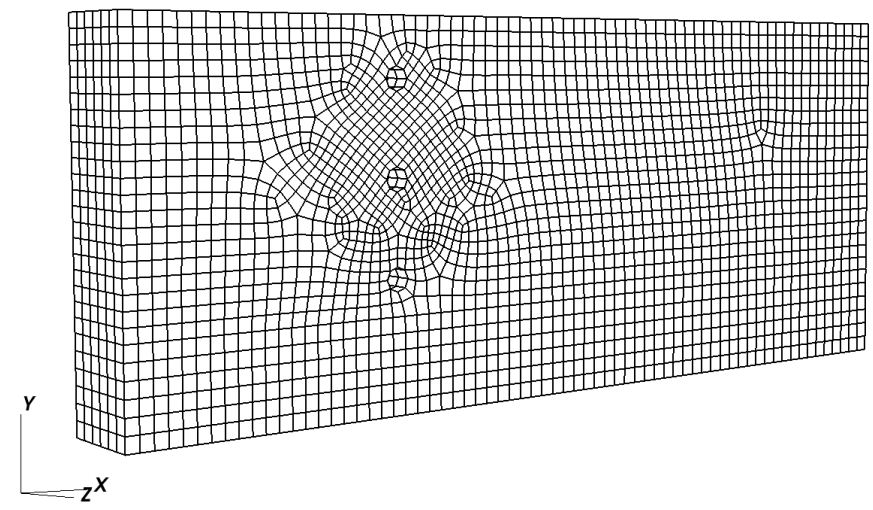

(b) Finite element mesh used in computations

Figure 14: Illustration of geometry, boundary conditions and the finite element mesh used in computations for the example problem of notched beam with holes. 


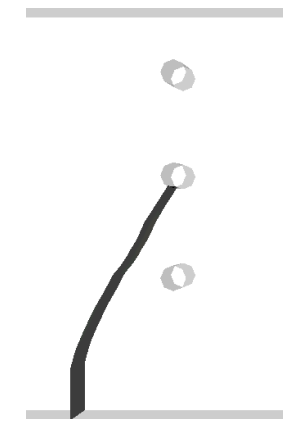

(a) Predicted crack path: case 1
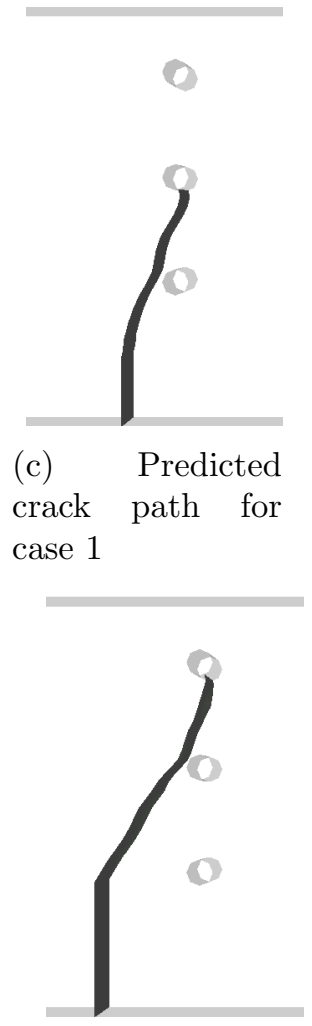

(e) Predicted crack path: case 3

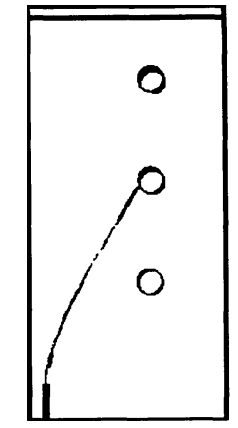

(b) Observed crack path: case 1

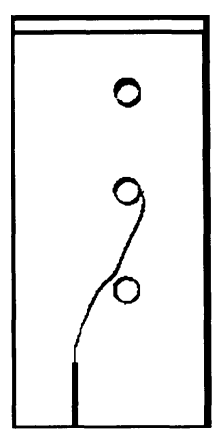

(d) Observed crack path: case 2

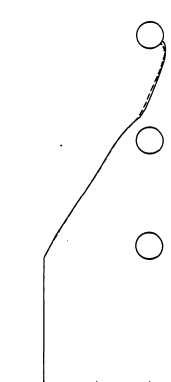

(f) Observed crack path: case 3

Figure 15: A comparison of predicted and observed crack trajectories for the notched beam with holes problem. On left, we plot the predicted crack trajectories from the proposed local crack-tracking approach for the three cases investigated experimentally by Ingraffea and Grigoriu [51]. On right, we plot the digitized photographs of the experimentally observed crack path. 

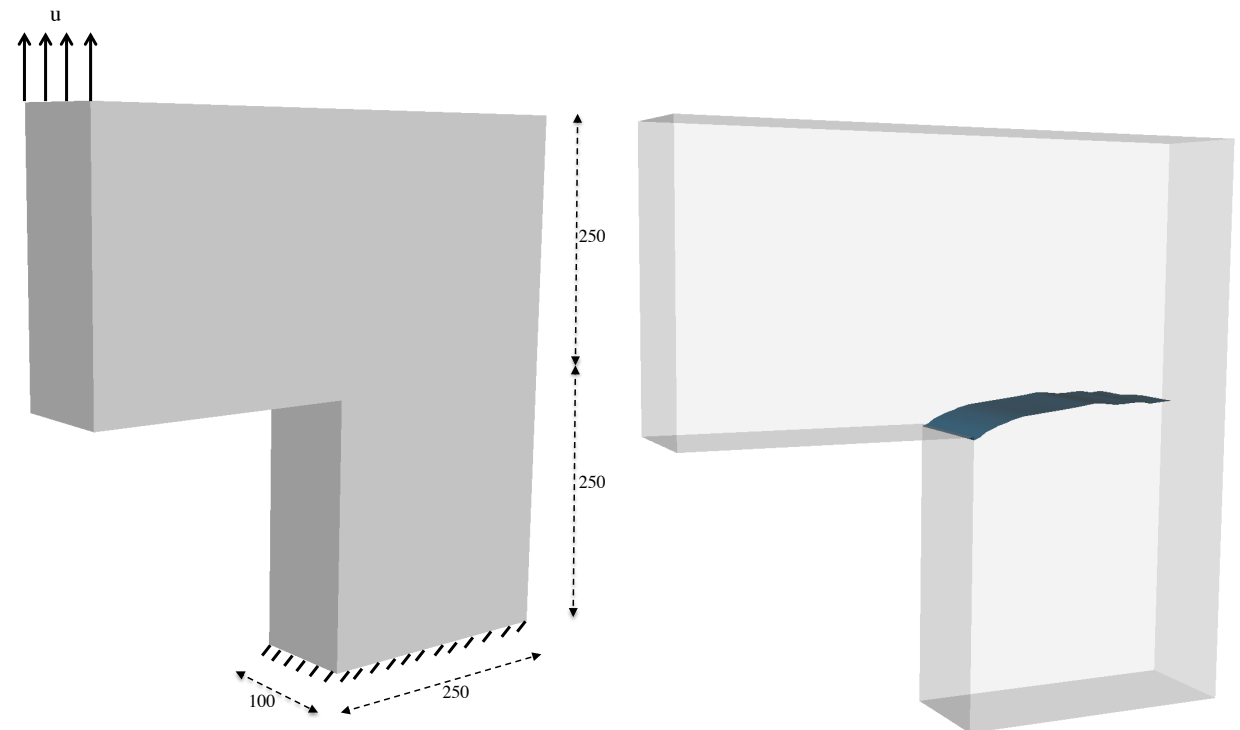

(a) Problem geometry and boundary (b) Predicted crack path from the proconditions posed local crack-tracking approach

Figure 16: Figure illustrating the geometry and loading conditions for the L-shaped panel (on left) and the crack path predicted by the proposed local crack-tracking approach at an applied displacement of $u=0.2 \mathrm{~mm}$ (on right) 


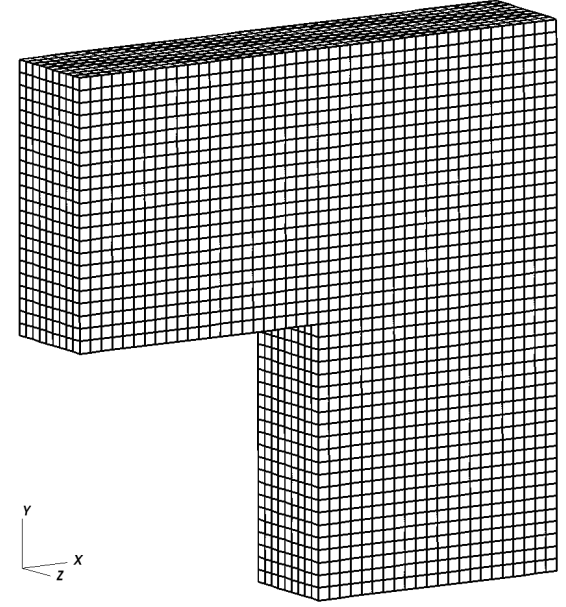

(a) Finite element mesh used for the L-shaped panel benchmark

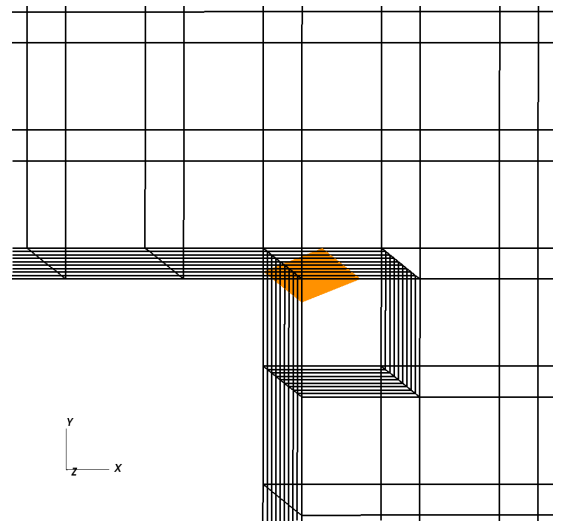

(b) A zoom of the mesh at the reentrant corner. The orange planar surface is the initial flaw introduced in the elements at the re-entrant corner

Figure 17: Figure illustrating the finite element mesh used for the L-shaped panel test (on left) and the extent and orientation of the intially introduced flaw (on right) 


\subsection{L-shaped panel}

We now model an L-shaped panel previously investigated experimentally in Winkler et al. [57] and reproduced numerically in several two and threedimensional studies since $[28,58,30,33]$. The geometry and the loading conditions are shown in Figure 16(a). We use a structured mesh of 13086 trilinear hexahedral elements (Figure 17). Consistent with earlier studies, we use a Young's modulus of $E=25.84 \mathrm{GPa}$ and a Poisson's ratio of $\nu=0.18$. We use a failure stress value of $\sigma_{f}=3 \mathrm{MPa}$. As we do not allow for crack initiation, a flaw (the size of one finite element) is introduced in the elements at the re-entrant corner as shown in Figure 17.

We apply the boundary conditions incrementally via displacement control, with the nodes at the indicated boundary displaced by $u=0.02 \mathrm{~mm}$ in each load step. The resulting crack-path is shown in Figure 16(b) at an applied displacement of $u=0.2 \mathrm{~mm}$. The crack initially curves upward before subsequently straightening as it approaches the right edge. The predicted crack path seems to be in good agreement with earlier studies $[28,30,58]$.

\subsection{Three-point bending test with an initially skewed notch}

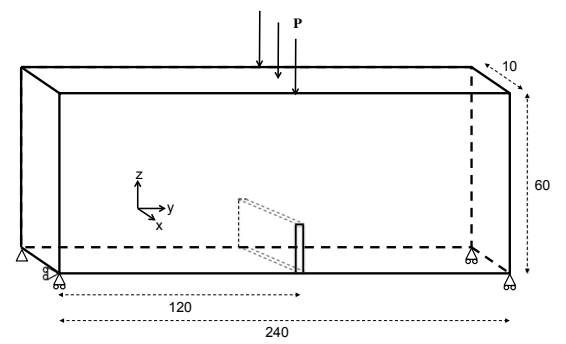

(a) Geometry and loading conditions for the three point bending problem with an initially skewed notch

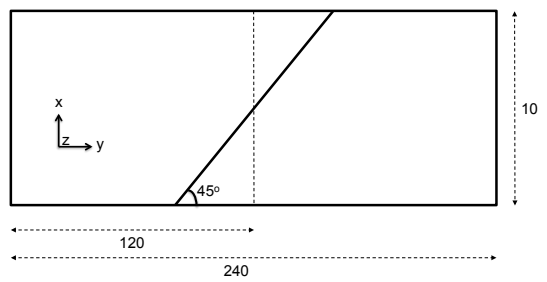

(b) Top view of the notch

Figure 18: Problem set-up and boundary conditions for the three point bending problem with an initially skewed notch

As a final example, we consider a three-point bending specimen with an initial notch at a 45 degree inclination to the plane of the cross section of the beam (see Figure 18). This problem has been investigated experimentally as well as in several computational studies before [59, 17, 58]. This example serves as a good benchmark to investigate non-planar crack growth as the 


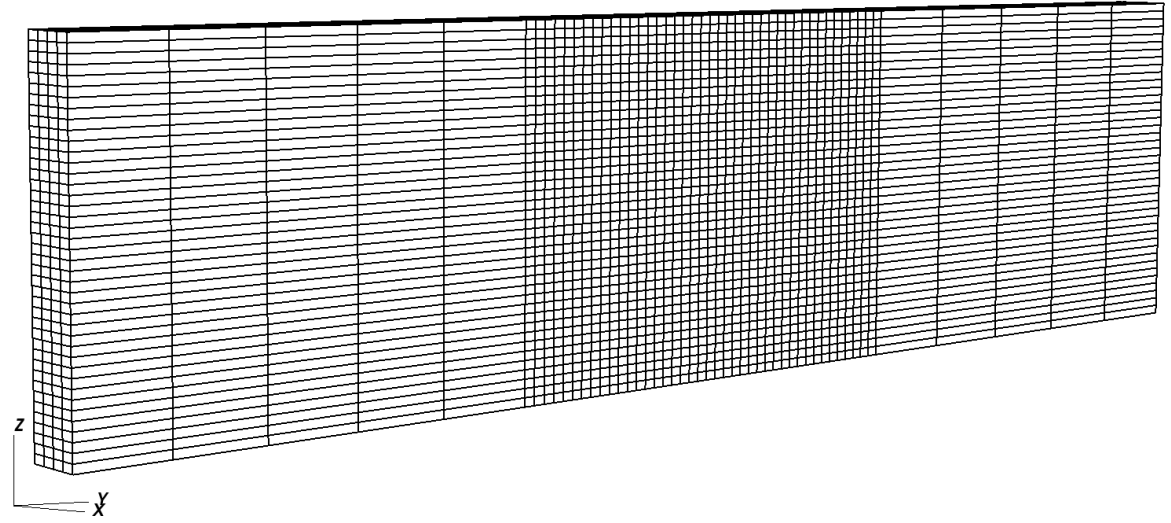

Figure 19: Finite element mesh used in computations for the crack twisting benchmark.

crack is expected to gradually twist around the direction of propagation and align itself with a direction consistent with the mode I loading conditions.

Consistent with earlier studies, we choose a Young's modulus of $E=2.8$ GPa, and Poisson's ratio of $\nu=0.38$. A failure stress value of $\sigma_{f}=7 \mathrm{MPa}$ is used. The geometry and boundary conditions are described in Figure 18. We use a structured mesh of 8624 trilinear hexahedral elements as shown in Figure 19. The value of the thermal field $\theta$ in the nodes of the elements cut by the pre-specified notch is initialized as a signed-distance to the crack such that the iso-surface $\theta=0$ tracks the crack. In order to compare, the predicted crack trajectory and the experimentally observed crack trajectory are plotted side-by-side in Figure 20. As seen from Figure 20, the predicted and observed crack trajectories are in good agreement. We also plot the top-view of the crack surface at different load steps in Figure 21 to better highlight the twisting behavior exhibited by the crack as it propagates.

\section{Conclusion}

We presented a local, implicit crack-tracking strategy to model embedded failure surfaces in three-dimensions. The presented approach relies on the solution of a Laplace equation with anisotropic conductivity. The equation is solved only in the elements just ahead of the crack front to yield a nodal value of a sign-distance field that is used to track the crack. The results demonstrate that unlike the global crack-tracking approach described in Oliver et al. (Int. J. Numer. Anal. Meth. Geomech., 2004; 28: 609-632), the proposed 


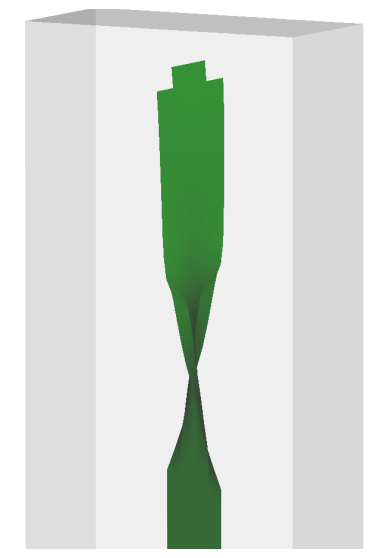

(a) Predicted failure surface from the proposed local crack-tracking approach

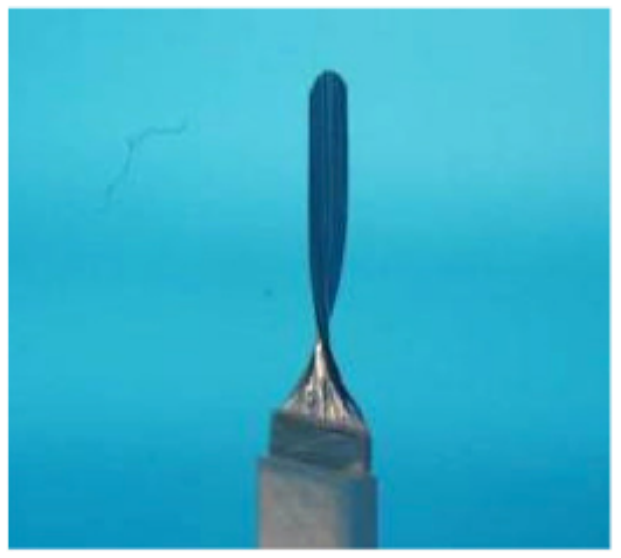

(b) Experimentally observed failure surface from Citarella and Buchholz [59]

Figure 20: A comparison between the predicted crack trajectories from the proposed local crack-tracking method and experimentally observed failure surfaces for the three point bending specimen with an initially skewed notch.

approach does not suffer from numerical sensitivity to the Dirichlet boundary conditions for the crack-tracking equation. In particular, in presence of closely interacting fractures, the performance of the proposed approach is found to be more robust. In addition, due to its local nature, the algorithm also provides an added benefit in terms of computational efficiency and a potential means to treat fracture intersections. We demonstrated through several numerical examples that the proposed algorithm can be reliably used to simulate crack propagation in 3D. Going forward, extending the proposed method to handle fracture intersections is of interest.

\section{Acknowledgements}

Chandrasekhar Annavarapu would like to thank Dr. Joshua A. White at Lawrence Livermore National Laboratory, USA for helpful discussions and comments. This document was prepared by LLNL under Contract DE-AC5207NA27344 and the document has been released to external audience under release number: LLNL-JRNL-696164. The financial support by the Laboratory Directed Research and Development program for the project \#15-ERD010 is gratefully acknowledged. 


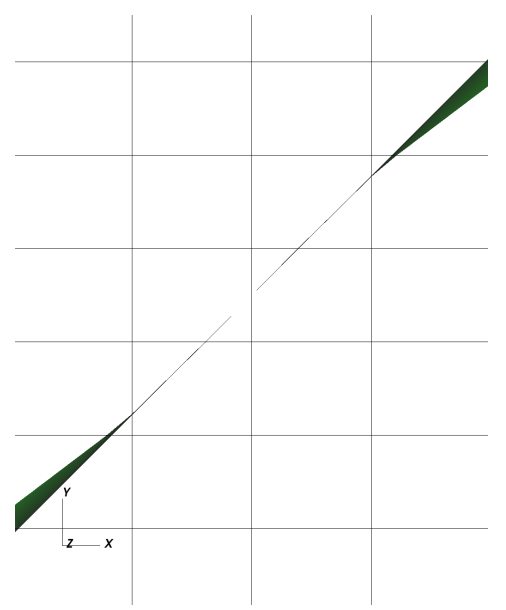

(a) Top view of the failure surface at a pseudo-time step $t=3$

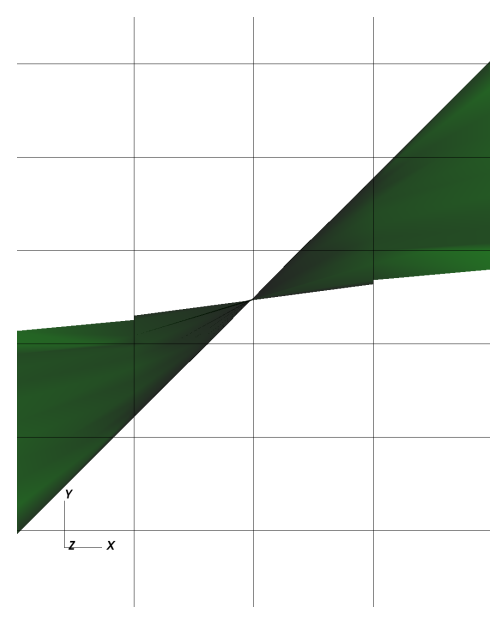

(c) Top view of the failure surface at pseudo-time step $t=13$

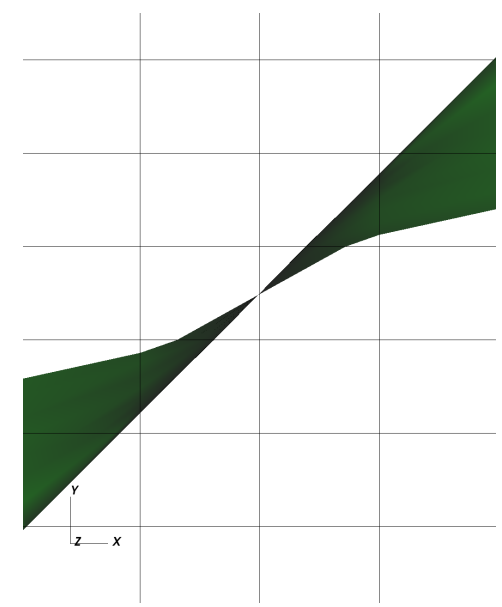

(b) Top view of the failure surface at pseudo-time step $t=9$

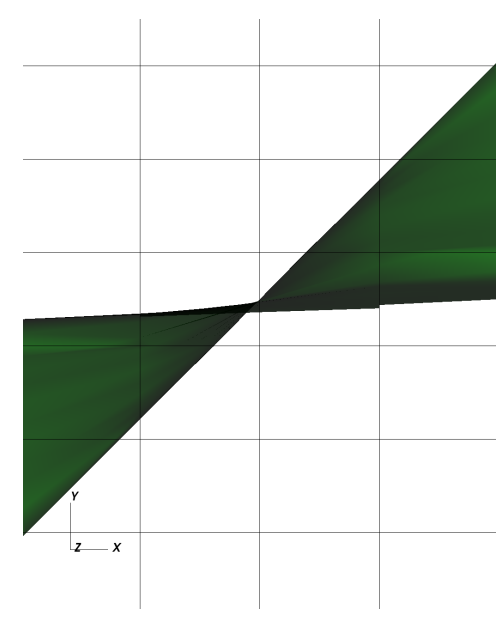

(d) Top view of the failure surface at pseudo-time step $t=20$

Figure 21: Top view of the failure surfaces observed at different load steps. It can be clearly observed that the crack twists to align itself with mode I loading conditions. 


\section{References}

[1] N. Moës, J. E. Dolbow, T. Belytschko, A finite element method for crack growth without remeshing, Int. J. Numer. Meth. Engng. 46 (1) (1999) $131-150$.

[2] C. Duarte, I. B. ska, J. Oden, Generalized finite element methods for three-dimensional structural mechanics problems, Computers and Structures 77 (2) (2000) $215-232$.

[3] A. Hansbo, P. Hansbo, A finite element method for the simulation of strong and weak discontinuities in solid mechanics, Comput. Methods Appl. Mech. Engrg. 193 (33-35) (2004) 3523 - 3540.

[4] A. Hansbo, P. Hansbo, An unfitted finite element method, based on Nitsche's method, for elliptic interface problems, Comput. Methods Appl. Mech. Engrg. 191 (47-48) (2002) 5537-5552.

[5] M. Ortiz, Y. Leroy, A. Needleman, A finite element method for localized failure analysis, Computer Methods in Applied Mechanics and Engineering 61 (2) (1987) $189-214$.

[6] J. C. Simo, J. Oliver, F. Armero, An analysis of strong discontinuities induced by strain-softening in rate-independent inelastic solids, Computational Mechanics 12 (5) (1993) 277-296.

[7] J. Oliver, Modelling strong discontinuities in solid mechanics via strain softening constitutive equations. part 1: Fundamentals, International Journal for Numerical Methods in Engineering 39 (21) (1996) 35753600 .

[8] J. Oliver, Modelling strong discontinuities in solid mechanics via strain softening constitutive equations. part 2: Numerical simulation, International Journal for Numerical Methods in Engineering 39 (21) (1996) 3601-3623.

[9] X.-P. Xu, A. Needleman, Numerical simulations of fast crack growth in brittle solids, Journal of the Mechanics and Physics of Solids 42 (9) (1994) $1397-1434$. 
[10] G. Camacho, M. Ortiz, Computational modelling of impact damage in brittle materials, International Journal of Solids and Structures 33 (20) (1996) $2899-2938$.

[11] P. M. A. Areias, T. Belytschko, Analysis of three-dimensional crack initiation and propagation using the extended finite element method, International Journal for Numerical Methods in Engineering 63 (5) (2005) 760-788.

[12] T. C. Gasser, G. A. Holzapfel, Modeling 3d crack propagation in unreinforced concrete using pufem, Computer Methods in Applied Mechanics and Engineering 194 (25-26) (2005) 2859-2896.

[13] C. Duarte, O. Hamzeh, T. Liszka, W. Tworzydlo, A generalized finite element method for the simulation of three-dimensional dynamic crack propagation, Computer Methods in Applied Mechanics and Engineering 190 (15-17) (2001) 2227-2262.

[14] J. P. Pereira, C. A. Duarte, D. Guoy, X. Jiao, hp-generalized fem and crack surface representation for non-planar 3-d cracks, International Journal for Numerical Methods in Engineering 77 (5) (2009) 601-633.

[15] J. P. Pereira, C. A. Duarte, X. Jiao, Three-dimensional crack growth with hp-generalized finite element and face offsetting methods, Computational Mechanics 46 (3) (2010) 431-453.

[16] J. Garzon, P. O’Hara, C. A. Duarte, W. G. Buttlar, Improvements of explicit crack surface representation and update within the generalized finite element method with application to three-dimensional crack coalescence, International Journal for Numerical Methods in Engineering 97 (4) (2014) 231-273.

[17] N. Moës, A. Gravouil, T. Belytschko, Non-planar 3d crack growth by the extended finite element and level sets?part i: Mechanical model, International Journal for Numerical Methods in Engineering 53 (11) (2002) 2549-2568.

[18] A. Gravouil, N. Moës, T. Belytschko, Non-planar 3d crack growth by the extended finite element and level sets?part ii: Level set update, International Journal for Numerical Methods in Engineering 53 (11) (2002) 2569-2586. 
[19] N. Sukumar, D. Chopp, B. Moran, Extended finite element method and fast marching method for three-dimensional fatigue crack propagation, Engineering Fracture Mechanics 70 (1) (2003) 29-48.

[20] N. Sukumar, D. L. Chopp, E. Béchet, N. Moës, Three-dimensional non-planar crack growth by a coupled extended finite element and fast marching method, International Journal for Numerical Methods in Engineering 76 (5) (2008) 727-748.

[21] M. Duflot, A study of the representation of cracks with level sets, International Journal for Numerical Methods in Engineering 70 (11) (2007) $1261-1302$.

[22] D. Colombo, P. Massin, Fast and robust level set update for 3d nonplanar x-fem crack propagation modelling, Computer Methods in Applied Mechanics and Engineering 200 (25-28) (2011) 2160-2180.

[23] T.-P. Fries, M. Baydoun, Crack propagation with the extended finite element method and a hybrid explicit-implicit crack description, International Journal for Numerical Methods in Engineering 89 (12) (2012) $1527-1558$.

[24] J. Oliver, A. E. Huespe, E. Samaniego, E. W. V. Chaves, Continuum approach to the numerical simulation of material failure in concrete, Int. J. Numer. Anal. Meth. Geomech. 28 (7-8) (2004) 609-632. doi:10.1002/nag.365.

URL http://dx.doi.org/10.1002/nag. 365

[25] M. Cervera, M. Chiumenti, Mesh objective tensile cracking via a local continuum damage model and a crack tracking technique, Computer Methods in Applied Mechanics and Engineering 196 (1-3) (2006) 304320 .

[26] C. Feist, G. Hofstetter, Three-dimensional fracture simulations based on the sda, International Journal for Numerical and Analytical Methods in Geomechanics 31 (2) (2007) 189-212.

[27] F. Armero, J. Kim, Three-dimensional finite elements with embedded strong discontinuities to model material failure in the infinitesimal range, International Journal for Numerical Methods in Engineering 91 (12) (2012) 1291-1330. 
[28] C. Linder, X. Zhang, A marching cubes based failure surface propagation concept for three-dimensional finite elements with non-planar embedded strong discontinuities of higher-order kinematics, International Journal for Numerical Methods in Engineering 96 (6) (2013) 339-372.

[29] C. Linder, X. Zhang, Three-dimensional finite elements with embedded strong discontinuities to model failure in electromechanical coupled materials, Computer Methods in Applied Mechanics and Engineering 273 (2014) 143-160.

[30] P. Jäger, P. Steinmann, E. Kuhl, Modeling three-dimensional crack propagation-a comparison of crack path tracking strategies, International Journal for Numerical Methods in Engineering 76 (9) (2008) 13281352.

[31] P. Jäger, P. Steinmann, E. Kuhl, On local tracking algorithms for the simulation of three-dimensional discontinuities, Computational Mechanics 42 (3) (2008) 395-406.

[32] P. Jäger, P. Steinmann, E. Kuhl, Towards the treatment of boundary conditions for global crack path tracking in three-dimensional brittle fracture, Computational Mechanics 45 (1) (2009) 91-107.

[33] P. Dumstorff, G. Meschke, Crack propagation criteria in the framework of $\mathrm{x}$-fem-based structural analyses, International Journal for Numerical and Analytical Methods in Geomechanics 31 (2) (2007) 239-259.

[34] G. Meschke, P. Dumstorff, Energy-based modeling of cohesive and cohesionless cracks via $\mathrm{x}$-fem, Computer Methods in Applied Mechanics and Engineering 196 (21-24) (2007) 2338-2357.

[35] J. Mergheim, E. Kuhl, P. Steinmann, A finite element method for the computational modelling of cohesive cracks, International Journal for Numerical Methods in Engineering 63 (2) (2005) 276-289.

[36] J.-H. Song, P. M. A. Areias, T. Belytschko, A method for dynamic crack and shear band propagation with phantom nodes, International Journal for Numerical Methods in Engineering 67 (6) (2006) 868-893. 
[37] G. Ventura, On the elimination of quadrature subcells for discontinuous functions in the extended finite-element method, International Journal for Numerical Methods in Engineering 66 (5) (2006) 761-795.

[38] Y. Sudhakar, W. A. Wall, Quadrature schemes for arbitrary convex/concave volumes and integration of weak form in enriched partition of unity methods, Computer Methods in Applied Mechanics and Engineering 258 (2013) 39-54.

[39] Y. Sudhakar, J. M. de Almeida, W. A. Wall, An accurate, robust, and easy-to-implement method for integration over arbitrary polyhedra: Application to embedded interface methods, Journal of Computational Physics 273 (2014) 393-415.

[40] S. Mousavi, N. Sukumar, Generalized gaussian quadrature rules for discontinuities and crack singularities in the extended finite element method, Computer Methods in Applied Mechanics and Engineering 199 (49-52) (2010) 3237-3249.

[41] G. N. Wells, L. J. Sluys, A new method for modelling cohesive cracks using finite elements, International Journal for Numerical Methods in Engineering 50 (12) (2001) 2667-2682.

[42] R. Settgast, P. Fu, S. Walsh, J. White, C. Annavarapu, F. Ryerson, A fully coupled method for massively parallel simulation of hydraulically driven fractures in 3-dimensions, International Journal for Numerical and Analytical Methods in Geomechanics article in press, doi:10.1002/nag.2557.

[43] R. Settgast, S. Johnson, P. Fu, S. Walsh, C. Annavarapu, Y. Hao, J. White, F. Ryerson, Geos: A framework for massively parallel multiphysics simulations. theory and implementation, Tech. rep., LLNL Technical Report 654611 (2014).

[44] C. Annavarapu, R. R. Settgast, S. M. Johnson, P. Fu, E. B. Herbold, A weighted nitsche stabilized method for small-sliding contact on frictional surfaces, Computer Methods in Applied Mechanics and Engineering 283 (2015) $763-781$.

[45] S. Melin, Why do cracks avoid each other?, International Journal of Fracture 23 (1) (1983) 37-45. 
[46] Y. Sumi, Z. Wang, A finite-element simulation method for a system of growing cracks in a heterogeneous material, Mechanics of Materials 28 (1-4) (1998) 197-206.

[47] N. Moës, C. Stolz, P.-E. Bernard, N. Chevaugeon, A level set based model for damage growth: The thick level set approach, International Journal for Numerical Methods in Engineering 86 (3) (2011) 358-380.

[48] J.-C. Sempere, K. C. Macdonald, Overlapping spreading centers: Implications from crack growth simulation by the displacement discontinuity method, Tectonics 5 (1) (1986) 151-163.

[49] M. Swain, J. Hagan, Some observations of overlapping interacting cracks, Engineering Fracture Mechanics 10 (2) (1978) 299 - 304.

[50] J. E. Olson, S. E. Laubach, R. H. Lander, Natural fracture characterization in tight gas sandstones: Integrating mechanics and diagenesis, AAPG bulletin 93 (11) (2009) 1535-1549.

[51] A. R. Ingraffea, M. Grigoriu, Probabilistic fracture mechanics: A validation of predictive capability, Tech. rep., DTIC Document (1990).

[52] T. Bittencourt, P. Wawrzynek, A. Ingraffea, J. Sousa, Quasi-automatic simulation of crack propagation for $2 \mathrm{~d}$ lefm problems, Engineering Fracture Mechanics 55 (2) (1996) 321-334.

[53] A. Mesgarnejad, B. Bourdin, M. Khonsari, Validation simulations for the variational approach to fracture, Computer Methods in Applied Mechanics and Engineering 290 (2015) 420-437.

[54] C. Miehe, E. Gürses, A robust algorithm for configurational-force-driven brittle crack propagation with r-adaptive mesh alignment, International Journal for Numerical Methods in Engineering 72 (2) (2007) 127-155.

[55] C. Miehe, M. Hofacker, F. Welschinger, A phase field model for rateindependent crack propagation: Robust algorithmic implementation based on operator splits, Computer Methods in Applied Mechanics and Engineering 199 (45-48) (2010) 2765-2778.

[56] T. Blacker, W. Bohnhoff, T. Edwards, CUBIT mesh generation environment. Volume 1: Users manual, 1994. 
[57] B. J. Winkler, Traglastuntersuchungen von unbewehrten und bewehrten Betonstrukturen auf der Grundlage eines objektiven Werkstoffgesetzes für Beton, Ph. D. Thesis, Innsbruck University Press, 2001.

[58] G. Ferté, P. Massin, N. Moës, 3d crack propagation with cohesive elements in the extended finite element method, Computer Methods in Applied Mechanics and Engineering 300 (2016) 347-374.

[59] R. Citarella, F.-G. Buchholz, Comparison of crack growth simulation by dbem and fem for sen-specimens undergoing torsion or bending loading, Engineering Fracture Mechanics 75 (3-4) (2008) 489 - 509. 